\title{
Interval propagation and search on directed acyclic graphs for numerical constraint solving
}

\author{
Xuan-Ha Vu • Hermann Schichl • \\ Djamila Sam-Haroud
}

Received: 20 November 2007 / Accepted: 2 December 2008 / Published online: 24 December 2008

(C) Springer Science+Business Media, LLC. 2008

\begin{abstract}
The fundamentals of interval analysis on directed acyclic graphs (DAGs) for global optimization and constraint propagation have recently been proposed in Schichl and Neumaier (J. Global Optim. 33, 541-562, 2005). For representing numerical problems, the authors use DAGs whose nodes are subexpressions and whose directed edges are computational flows. Compared to tree-based representations [Benhamou et al. Proceedings of the International Conference on Logic Programming (ICLP'99), pp. 230-244. Las Cruces, USA (1999)], DAGs offer the essential advantage of more accurately handling the influence of subexpressions shared by several constraints on the overall system during propagation. In this paper we show how interval constraint propagation and search on DAGs can be made practical and efficient by: (1) flexibly choosing the nodes on which propagations must be performed, and (2) working with partial subgraphs of the initial DAG rather than with the entire graph. We propose a new interval constraint propagation technique which exploits the influence of subexpressions on all the constraints together rather than on individual constraints. We then show how the new propagation technique can be integrated into branch-and-prune search to solve numerical constraint satisfaction problems. This algorithm is able to outperform its obvious contenders, as shown by the experiments.
\end{abstract}

Keywords Interval constraint propagation - Directed acyclic graphs $\cdot$ Branch and prune

A short version of this paper has been published in [27].

X.-H. Vu

Cork Constraint Computation Centre, University College Cork, 14 Washington Street West, Cork, Ireland e-mail: ha.vu@4c.ucc.ie

H. Schichl

Faculty of Mathematics, University of Vienna, Nordbergstr. 15, 1090 Wien, Austria

e-mail: hermann.schichl@univie.ac.at

D. Sam-Haroud $(\bowtie)$

Artificial Intelligence Laboratory, Ecole Polytechnique Fédérale de Lausanne (EPFL), Batiment IN, Station 14, 1015 Lausanne, Switzerland

e-mail: jamila.sam@epfl.ch 


\section{Introduction}

A constraint satisfaction problem (CSP) consists of a finite set of constraints specifying which combinations of values from given domains of variables are admitted. A CSP is said to be numerical if its domains are continuous. Numerical CSPs, such as systems of nonlinear equations and inequalities, arise in many applications and form a difficult problem class since they are NP-hard. In practice, numerical constraints are usually expressed in factorable form which means that they are composed of elementary operators or functions (e.g.,,$+ *$, $\div, \sqrt{ }$ and $\sin$ ) and standard relations (e.g., $\leq,<, \neq,>$, and $\geq$ ). Many solution techniques exploit the factorability of such numerical constraints to efficiently solve numerical CSPs. To achieve full mathematical rigor when performing operations on floating-point numbers, most techniques are based on interval arithmetic or its variants.

The most commonly used complete strategy for finding the solutions of a numerical CSP is branch-and-prune, which interleaves branching steps with pruning steps. Roughly speaking, a branching step divides the problem into subproblems whose union is equivalent to the initial one in term of the solution set, and a pruning step reduces/simplifies the problem in some measure. The most well-known pruning technique is domain reduction, which reduces the domains of variables without discarding any solution of the problem.

Over the last 20 years, many domain reduction techniques based on interval arithmetic have been devised. In particular, an interesting approach in constraint programming, called interval constraint propagation, was developed in the 1990s (see [2,3,24] and [9]). This approach combines constraint propagation techniques, as defined in artificial intelligence, with interval-analytic methods. The algorithm HC4 [5] is one of the most prominent representatives of this family of domain reduction techniques. In HC4, each individual constraint is represented by a tree whose nodes and edges stand respectively for subexpressions and computational flows. Each node of the tree is associated with the (possible) range of the corresponding subexpression.

In order to reduce the variables' domains of a given constraint the technique recursively performs forward evaluations and then backward projections on the whole tree representing the constraint. These two steps compute the ranges of nodes based on the ranges of their children's and parents' respectively. When several constraints are involved, HC4 performs forward evaluations and backward projections individually on each constraint, and then propagates the reduction of the variables' domains from tree to tree by using a variant of arc consistency, AC3 [13].

The fact that each constraint is propagated individually is one of the main limitations of this approach. The effects of the common subexpressions, shared by several constraints, is only roughly taken into account.

Recently, a fundamental framework for interval analysis on directed acyclic graphs (DAGs) has been proposed by [21] which overcomes this limitation. The authors suggested to replace trees with DAGs and showed how to perform forward evaluations and backward projections using this particular representation. The shift to DAGs potentially reduces the amount of computation on common subexpressions shared by constraints, and explicitly relates constraints to constraints in the natural way they are composed, thus enhancing the constraint propagation process.

The constraint propagation technique proposed in [21] is a direct generalization of HC4 in the sense that all the nodes of the DAG are forward evaluated then backward projected at once. In practice, and as the problems grow large, situations often occur where only a small number of nodes is worth considering for forward or backward inference as the other nodes leave the domain ranges unchanged after computation. 
This paper builds on this idea and presents a new constraint propagation technique following the DAG-based framework [21]. The contribution is twofold. Firstly, we show how the DAG-based framework can be made efficient and practical by adaptively performing forward evaluations and backward projections on chosen nodes of a DAG (see Sects. 4, 4.3 and 5). In our approach, switching from evaluations to projections is made at the node level rather than at the tree or DAG level.

Secondly, we show how the new propagation technique can be integrated into a generic branch-and-prune search without the necessity to create multiple DAGs (see Sects. 4.3, 5 and 6). Our experiments carried out on impartially chosen benchmarks show that the new technique outperforms previously available propagation techniques by 1 to 2 orders of magnitude or more in speed, while being roughly the same quality with respect to enclosure properties (see Sect. 7).

The paper is organized as follows. Section 2 presents the necessary background and definitions, including the fundamentals of numerical constraint satisfaction (Sect. 2.1), and DAG representation of numerical CSPs (Sect. 2.2). Section 3 describes a slight modification to standard interval arithmetic that may reduce the amount of computation in constraint propagation. Forward evaluation and backward propagation on DAGs as well as the notion of partial DAG representation are presented in Sect. 4 and serve as basis for the core contributions of this paper (Sects. 5 and 6). Finally, Sect. 7 discusses the preliminary experimental results.

\section{Background and notation}

Recall that a factorable function is one, which can be expressed as a finite composition of pre-specified elementary operations, and that a constraint is called factorable if it involves only factorable functions.

In the composition of a factorable constraint, each constraint representing an elementary operation is called a primitive constraint.

A CSP solely consisting of factorable constraints is called factorable.

All of our work will be based on interval analysis. Extended introductions on the subject can be found in $[1,15,16]$, on interval methods for systems of equations in [17], on interval methods for optimization in [7], and on some recent applications of interval arithmetic in [9].

We will use the following notation: We write $\mathbb{R}_{\infty} \equiv \mathbb{R} \cup\{-\infty,+\infty\}$. The set of all closed intervals is denoted by $\mathbb{I}$ and the set of all intervals is denoted by $\mathbb{R}$. For a subset $S$ of $\mathbb{R}$ we denote the interval hull by $\square S$.

The lower bound of a real interval $\mathbf{x}$ is defined as inf( $(\mathbf{x})$, and the upper bound as $\sup (\mathbf{x})$. Let us denote $\underline{x}=\inf (\mathbf{x}) \in \mathbb{R}_{\infty}$ and $\bar{x}=\sup (\mathbf{x}) \in \mathbb{R}_{\infty}$. The midpoint will be written as $\operatorname{mid}(\mathbf{x})$, the radius as $\operatorname{rad}(\mathbf{x})$, and the width as $\mathrm{w}(\mathbf{x})$.

Let $f: D \subseteq \mathbb{R}^{n} \rightarrow \mathbb{R}^{m}$ be a factorable function with one of its realizations $\mathfrak{f}$ as arithmetic expression. The natural extension of $\mathfrak{f}$ is an interval function $\mathbf{f}_{\mathfrak{f}}: \mathbb{I} \mathbb{R}^{n} \rightarrow \mathbb{I} \mathbb{R}^{m}$ constructed from $\mathfrak{f}$ in which each real variable is replaced by an interval variable and each elementary real operation is replaced by the natural extension of this operation. It is easy to prove that $\mathbf{f}_{\mathfrak{f}}$ is an interval form of the function $f$. Therefore, it is called a natural interval form of $f$ and denoted by $\mathbf{f}$. Note that $\mathbf{f}$ indeed depends on the arithmetic expression used to realize $f$ (see e.g. [17, Chap. 1]), however, it is common usage to call every natural extension of $f$ the natural (interval) extension of $f$. For example, the natural interval form of the real function $f(x, y)=2 * x+x * y$ is the interval function $\mathbf{f}(\mathbf{x}, \mathbf{y})=2 * \mathbf{x}+\mathbf{x} * \mathbf{y}$. However, the same function $f$ can be (better) represented by the interval function $\mathbf{f}(\mathbf{x}, \mathbf{y})=\mathbf{x}(2+\mathbf{y})$. 
2.1 Numerical constraint satisfaction

\subsubsection{Numerical constraint satisfaction problems}

A constraint on a finite sequence of variables $\left(x_{1}, \ldots, x_{k}\right)$ taking values in respective domains $\left(D_{1}, \ldots, D_{k}\right)$ is a subset of the Cartesian product $D_{1} \times \cdots \times D_{k}$, where $k$ is a natural number, that is, in $\mathbb{N}$.

The concept of a constraint satisfaction problem is defined as follows.

Definition 1 A constraint satisfaction problem, abbreviated to CSP, is a triple $(\mathcal{V}, \mathcal{D}, \mathcal{C})$ in which $\mathcal{V}$ is a finite sequence of variables $\left(v_{1}, \ldots, v_{n}\right), \mathcal{D}$ is a finite sequence of the respective domains of the variables, and $\mathcal{C}$ is a finite set of constraints (each on a subsequence of $\mathcal{V}$ ). A solution of this problem is an assignment of values from $\mathcal{D}$ to $\mathcal{V}$ respectively such that all constraints in $\mathcal{C}$ are satisfied. The set of all solutions is called the solution set.

In this paper, we only focus on numerical CSPs defined as follows.

Definition 2 A numerical constraint is a constraint on a sequence of variables whose domains are continuous. A domain is called a continuous if its a real interval. If all the constraints of a CSP are numerical, this CSP is called a numerical constraint satisfaction problem (abbreviated to NCSP).

In practice, a NCSP can often be represented in the following form:

$$
f(x) \in \mathbf{b},
$$

where $x$ is a vector of $n$ real variables taking values in a box $\mathbf{x} \in \mathbb{I}^{n}, \mathbf{b} \equiv\left(\mathbf{b}_{1}, \mathbf{b}_{2}, \ldots, \mathbf{b}_{m}\right)^{\mathrm{T}}$ is an interval vector in $\mathbb{I}^{m}$, and $f=\left(f_{1}, f_{2}, \ldots, f_{m}\right)^{\mathrm{T}}$ is a factorable function from $D \subseteq \mathbb{R}^{n}$ to $\mathbb{R}^{m}$. For each $j=1, \ldots, n$, the interval $\mathbf{b}_{j}$ is called the constraint range of the constraint $f_{j}(x) \in \mathbf{b}_{j}$.

Since more than 30 years ago, constraint satisfaction techniques, such as arc consistency $[14,28,29]$ and path consistency [14], have been devised to solve CSPs with discrete domains. Those techniques perform reasoning procedures on constraints and explore the search space by intelligently enumerating solutions. In order to solve NCSPs by means of constraint satisfaction, continuous domains have often been converted into discrete domains by using progressive discretization techniques [12,19]. Later on, many mathematical computation techniques for continuous domains have been integrated into the framework of constraint satisfaction in order to solve NCSPs more efficiently. Nowadays, these techniques are often referred to as constraint programming, which implies the combination of computing and reasoning aspects.

Most techniques for solving NCSPs follow the branch-and-prune framework, which interleaves branching steps with pruning steps. A branching step divides a problem into subproblems whose union is equivalent to the initial problem in term of the solution set, and a pruning step reduces a problem. Pruning steps are usually performed by using domain reduction techniques, which reduce the domains of variables without discarding any solution of the problem. Inspired by the classical constraint satisfaction techniques, an interesting approach in constraint programming, called interval constraint propagation, was developed in the 1990s (see [2,3,24] and [9]). This approach combines constraint propagation techniques in constraint satisfaction with interval-analytic methods in mathematics. The idea is that one cannot exactly achieve consistency properties such as arc consistency for numerical constraints under floating-point number systems, therefore replaces the consistencies with 
relaxations that are tractable under floating-point number systems. For example, given a constraint $c$ on variables $\left(x_{1}, \ldots, x_{k}\right)$ with respective domains $\left(D_{1}, \ldots, D_{k}\right)$, arc consistency reduces each $D_{i}$ to the projection of $c$ on $x_{i}$, denoted $c\left[x_{i}\right]$. The interval variant of arc consistency only reduces each $D_{i}$ to the smallest union of intervals that contains $c\left[x_{i}\right]$. However, this is still intractable in practice. One may wish to replace it with a weaker property that each $D_{i}$ is the smallest interval containing $c\left[x_{i}\right]$. This introduces a new concept of consistency: hull consistency [2,3]. Other concepts of consistency such as $k \mathrm{~B}$-consistency [11] and box consistency [4] have also been introduced. In constraint programming, achieving those consistency properties has often been implemented by a search or interval constraint propagation technique in combination with mathematical tools such as interval arithmetic.

\subsubsection{Achieving hull consistency by constraint propagation}

Let be given a factorable numerical constraint and one of its compositions. In [2,3] the authors proposed to achieve hull consistency for the initial constraint by achieving hull-consistency for its primitive constraints in the given composition [5]. A faster propagation algorithm to achieve hull consistency for a single constraint was proposed in [5]. To reduce the domains of the variables of a number of constraints, the technique, called $\mathbf{H C 4}$, achieves hull consistency for individual constraints, and then propagates the reduction of the variables' domains from constraint to constraint by using a variant of arc consistency, AC3 [13].

To solve a NCSP of the form (1), the HC4 algorithm represents each constraint of the problem as a tree (defining a way to compose the constraint). Each node of the tree represents a primitive constraint. Each node $\mathbf{N}$ of the tree is associated with two intervals, called the forward and backward node ranges, denoted $\mathbf{N}^{\mathrm{f}}$ and $\mathbf{N}^{\mathrm{b}}$, respectively.

The exact value, hence the exact range, of the subexpression represented by a node must be contained in both of the two node ranges.

Example 1 The tree representation of the following NCSP is depicted in Fig. 1:

$\left\{\sqrt{x}+2 \sqrt{x y}+2 \sqrt{y} \leq 7,0 \leq x^{2} \sqrt{y}-2 x y+3 \sqrt{y} \leq 2, x \in[1,16], y \in[1,16]\right\}$.

The HC4 algorithm is presented as Algorithm 1. It invokes another algorithm, HC4revise, to achieve hull consistency for a constraint. HC4revise performs two main processes: recursive forward evaluation (RFE) and recursive backward projection (RBP). HC4revise is presented concisely in Algorithm 2, where $\mathcal{T}_{\mathbf{N}}$ denotes the tree rooted at node $\mathbf{N}$. At Line 1 of RBP, an elementary operation $\psi\left(\mathbf{N}_{1}, \ldots, \mathbf{N}_{q}\right)$ represented by node $\mathbf{N}$ defines a relation $\psi^{*}$ on the sequence $\left(\mathbf{N}, \mathbf{N}_{1}, \ldots, \mathbf{N}_{q}\right)$; where $\mathbf{N}, \mathbf{N}_{1}, \ldots, \mathbf{N}_{q}$ play the role of variables taking values in $\mathbf{N}^{\mathrm{b}}, \mathbf{N}_{1}^{\mathrm{f}}, \ldots, \mathbf{N}_{q}^{\mathrm{f}}$, respectively. Since $\psi$ is an elementary operation, $\psi^{*}$ is very simple and can be projected on its variables by using simple formulas in [5].

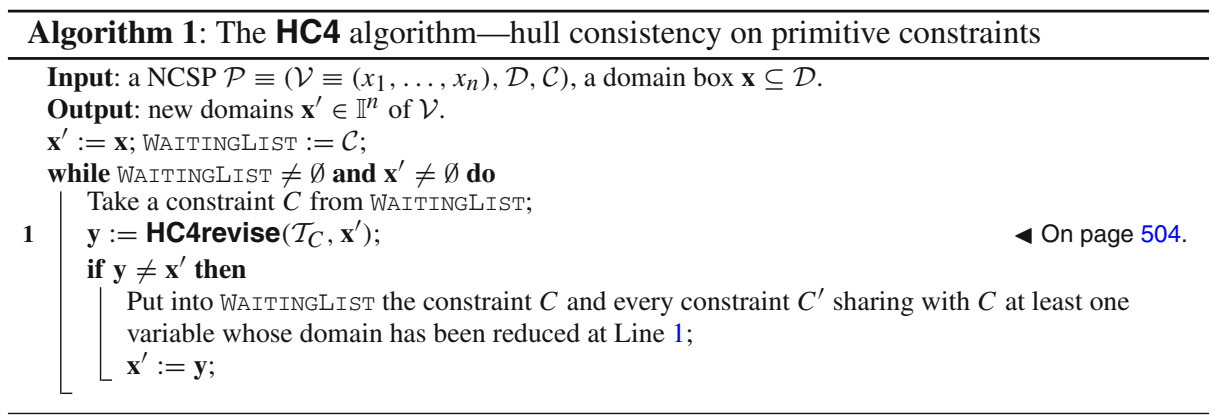




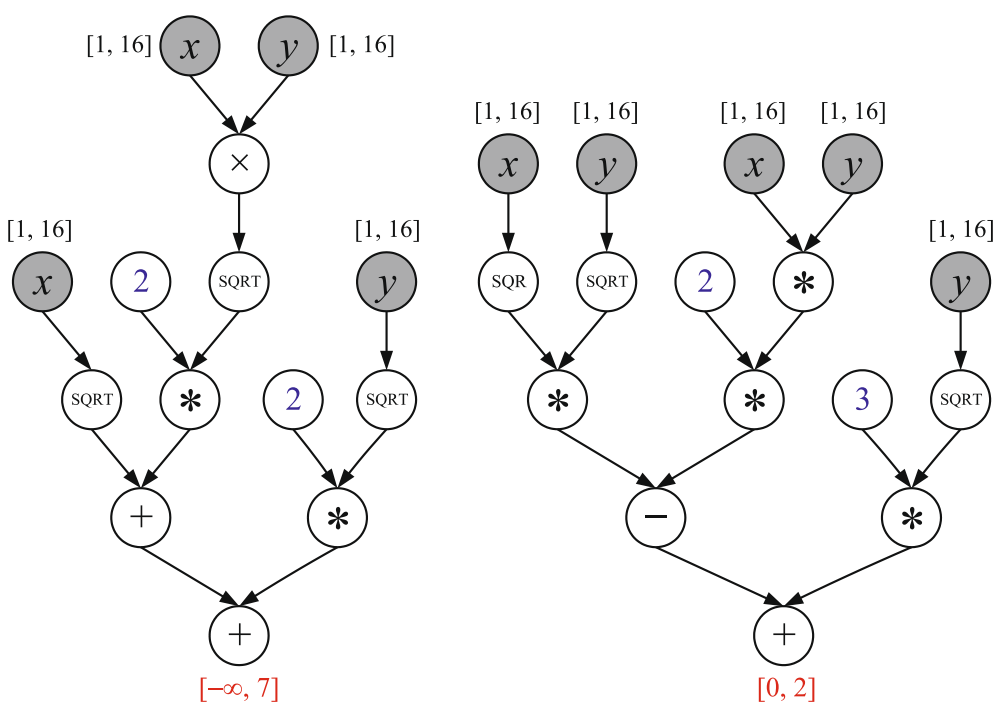

Fig. 1 The tree representation of the NCSP in Example 1
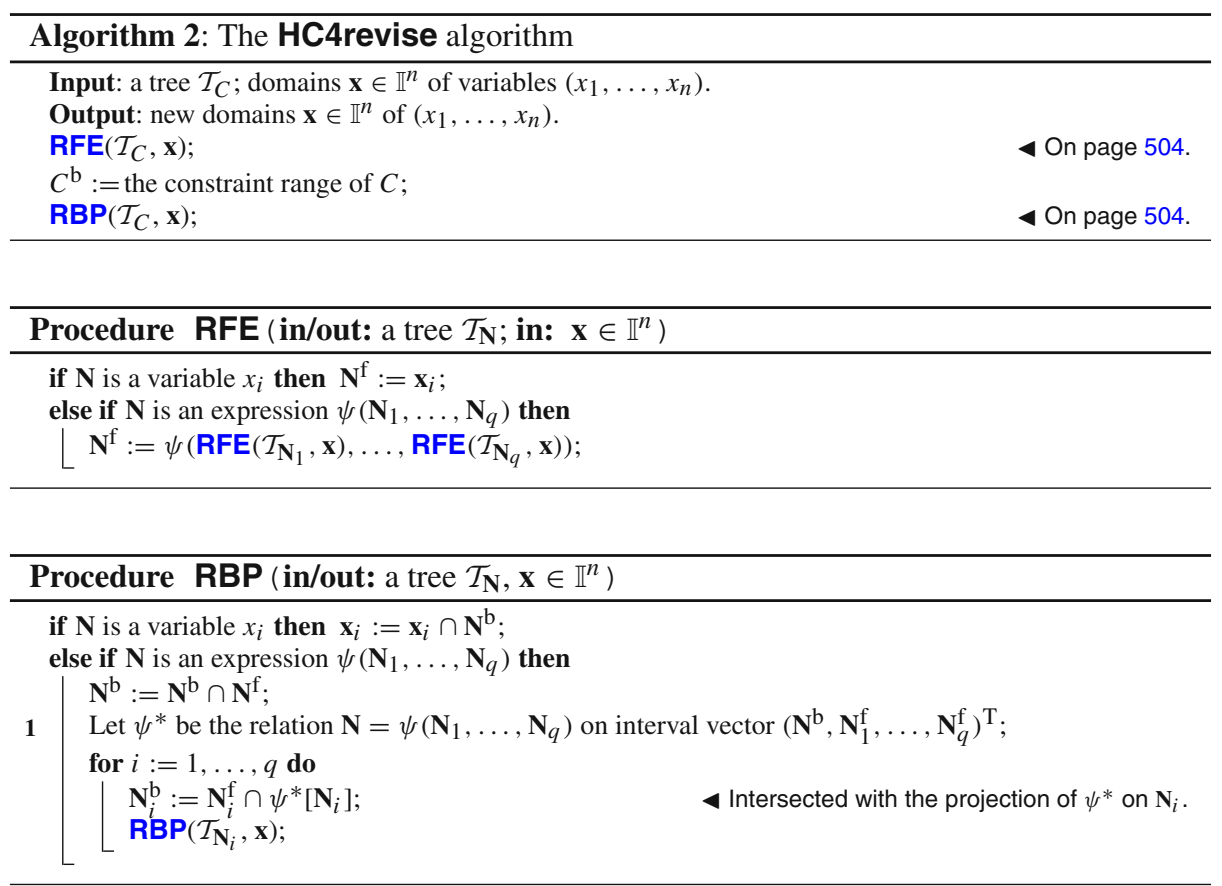

\subsection{DAG representations for numerical CSPs}

We use the concept of a DAG representation of a constraint system as in [21].

We recall the following fundamental result, which illustrates the precedence relationship of nodes in a directed acyclic multigraph. 
Theorem 1 For every directed acyclic multigraph $(V, E, f)$, there exists a total order $\preceq$ on the vertices $V$ such that for every $v \in V$ and every ancestor $u$ of $v$, we have $v \preceq u$.

Remark 1 Procedure NodeLevel on page 517, is a simple algorithm for assigning a level to each node such that any sorting in descending order of the obtained levels will result in a required order.

\subsubsection{DAG representation}

As proposed in [21], a directed acyclic multigraph with ordered edges, abbreviated to $D A G$, can be used to represent the factorable NCSP (1). Since the problem (1) is factorable, the function $f$ can be composed of a sequence of elementary operations/functions such as + , $*, /, \log$, exp, sqr, and sqrt. In this composition, each variable is represented by a leaf. Each elementary operation/function $\phi: D \subseteq \mathbb{R}^{k} \rightarrow \mathbb{R}$ that takes as input $k$ subexpressions $x_{1}, \ldots, x_{k}$ is represented by a node $\mathbf{N}$ with $k$ edges, each runs from the node representing $x_{i}$ to the node $\mathbf{N}$, where $1 \leq i \leq k$. These $k$ edges represent the computational flow in the natural composition of the operation $\phi$. The obtained representation is called the $D A G$ representation of the considered problem.

Notation 1 Each node $\mathbf{N}$ in the DAG representation is associated with an interval, denoted as $\tau_{\mathbf{N}}$ and called the node range of $\mathbf{N}$, in which the exact range of the associated subexpression must lie. $\mathbf{N}$ is also associated with a real variable, denoted by $\vartheta_{\mathbf{N}}$, that represents the value of the subexpression represented by $\mathbf{N}$.

For efficiency and compactness, the standard elementary operations in the DAG representation are replaced with more general operations. For example, multiple applications of binary elementary operations of the forms in $\{x+y, x-y, x+a, a+x, x-a, a-x, a x\}$ are replaced with a $k$-ary operation $a_{0}+a_{1} x_{1}+\cdots+a_{k} x_{k}$, which is interpreted as a $k$-ary operation + (see Fig. 2a), where $1 \leq k \in \mathbb{N}$. Similarly, multiple applications of the binary multiplication $x * y$ are replaced with a $k$-ary multiplication (or product) $a_{0} * x_{1} * \cdots * x_{k}$, which is interpreted as the $k$-ary operation $*$ (see Fig. 2b), where $2 \leq k \in \mathbb{N}$. In general, each edge of a DAG representation is associated with a respective coefficient of the operation represented by its target. When not specified in figures, this coefficient equals to 1 . The other constants involving an operation are stored at the node representing the operation (see Fig. 2). As a result, the DAG representation no longer have nodes representing constants as in the tree representation (see Sect. 2.1.2).

Much more detailed descriptions of DAG representations can be found in Sects. 4.1 and 5.3 of [20].

We need to use multigraphs, for efficiency, instead of simple graphs for DAG representations because some special operations can take the same input more than once. For example,

Fig. 2 A node and its computational flows in a DAG representation

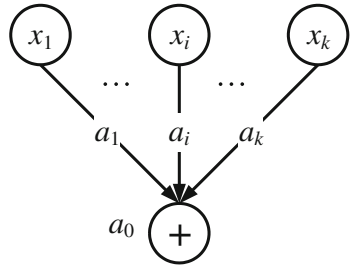

(a)

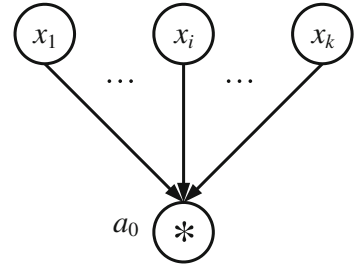

(b) 
the expression $x^{x}$ can be represented by the binary power operation $x^{y}$ without introducing a new unary operation $x^{x}$. In all cases, a normal directed acyclic graph is sufficient to represent a NCSP, provided that we introduce new elementary operations such as the unary operation $x^{x}$. The ordering of edges is needed for non-commutative operations like the division. For convenience, a ground node, called $\mathbf{G}$, is added to each DAG representation to be the parent of all nodes that represents the constraints. In fact, the ground node can be interpreted as the logical AND operation.

Example 2 Consider the following constraint system

$$
\left\{\begin{array}{l}
\sqrt{x}+2 \sqrt{x y}+2 \sqrt{y} \leq 7, \\
0 \leq x^{2} \sqrt{y}-2 x y+3 \sqrt{y} \leq 2, \\
x \in[1,16], y \in[1,16],
\end{array}\right.
$$

which can be written into the form (1) as follows

$$
\left\{\begin{array}{l}
\sqrt{x}+2 \sqrt{x y}+2 \sqrt{y} \in[-\infty, 7], \\
x^{2} \sqrt{y}-2 x y+3 \sqrt{y} \in[0,2], \\
x \in[1,16], y \in[1,16] .
\end{array}\right.
$$

The DAG representation of the constraint system (2) is depicted in Fig. 3. Two constraints of (2) are represented by two nodes $\mathbf{N}_{9}$ and $\mathbf{N}_{10}$. Two variables, $x$ and $y$, are represented by two nodes $\mathbf{N}_{1}$ and $\mathbf{N}_{2}$, respectively. The sequence $\left(\mathbf{N}_{1}, \mathbf{N}_{2}, \ldots, \mathbf{N}_{10}\right)$ of nodes given in Fig. 3 is an example of ordering as stated in Theorem 1.

For the same constraint system, the DAG representation is clearly more concise than the tree representation described in Sect. 2.1.2.

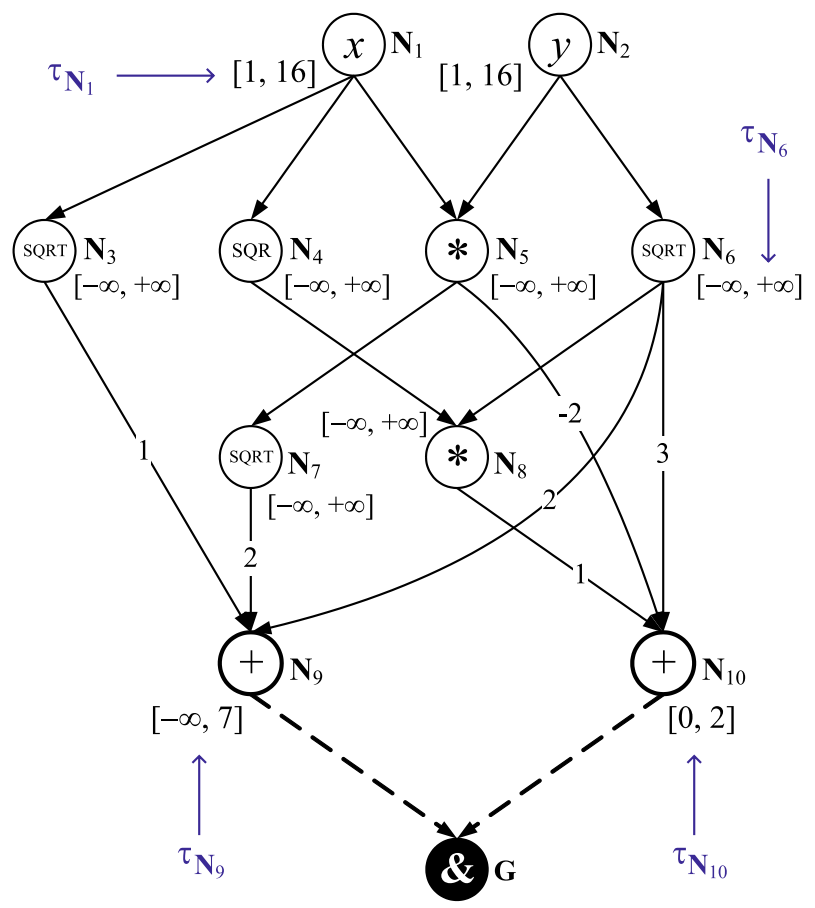

Fig. 3 The DAG representation of the constraint system (2) 


\section{Modification to standard interval arithmetic}

Expressions that are only defined on subsets of $\mathbb{R}^{n}$ are often encountered in practice. For example, a division by zero, such as $1 \div 0$, is not defined. Consequently, the division of two intervals is not defined in (standard) interval arithmetic when the denominator contains zero. In such cases, many implementations of interval arithmetic give, by convention, the universe interval $[-\infty, \infty]$ as result. This is an extension of (standard) interval arithmetic for all purposes, in order to conform to the inclusion property. If we use this implementation to evaluate the range of a function $f: D \subset \mathbb{R}^{n} \rightarrow \mathbb{R}^{m}$, we will often get unnecessarily overestimated bounds of the form $[-\infty, \infty]$ in case the denominators of the divisions in $f$ contain zero. In order to avoid such over-estimations, we have to extend functions depending on their use in specific computations. In this section, building on the concept of a multifunction, we propose a way to extend functions that are only defined on subsets of $\mathbb{R}^{n}$.

We do not use the concept of constraint sets (see e.g. [18]) here because they often provide overly pessimistic enclosures of the result of arithmetic operations. For example, the cset division is equivalent to $\div \mathbb{R}$, which is neither in forward mode nor in backward mode optimal.

\subsection{Extending domains of functions}

We start by recalling the definition of a multifunction from [23, p. 34].

Definition 3 (Multifunction) Let $X$ and $Y$ be two sets. A multifunction $F$ from $X$ to $Y$ (a relation on $X \times Y$ ), denoted as $F: X \rightarrow Y$, is a subset $F \subseteq X \times Y$. The inverse of $F$ is a multifunction $F^{-1}: Y \rightarrow X$ defined by the rule: $(y, x) \in F^{-1} \Leftrightarrow(x, y) \in F$. We define the values of $F$ at $x$ to be $F(x) \equiv\{y \in Y \mid(x, y) \in F\}$, and the fibers of $F$ for $y \in Y$ to be $F^{-1}(y) \equiv\{x \in X \mid(x, y) \in F\}$.

In Definition 3, if for some $x \in X$ there is no $y \in Y$ such that $(x, y) \in F$, we have that $F(x)=\emptyset$. From Definition 3 we can see that a function is, in fact, a special multifunction that is single-valued.

The concepts of image and inverse image (of a set) under a multifunction are similar to those for functions:

Definition 4 (Image, Inverse Image) Let $X$ and $Y$ be two sets, $F: X \rightarrow Y$ a multifunction. The image of a subset $A \subseteq X$ under $F$ is defined and denoted by

$$
F(A) \equiv \bigcup_{x \in A} F(x)=\left\{y \in F^{-1} \mid F^{-1}(y) \cap A \neq \emptyset\right\} .
$$

The inverse image of a subset $B \subseteq Y$ under $F$ is defined and denoted by

$$
F^{-1}(B) \equiv \bigcup_{y \in B} F^{-1}(y)=\{x \in F \mid F(x) \cap B \neq \emptyset\} .
$$

Next, we define a special class of multifunctions.

Definition 5 (Extended Function) Let $f$ be a function from a set $X$ to a set $Y, X^{\prime}$ a superset of $X$, and $Z$ a set of some subsets of $Y$ possibly including $\emptyset$. A Z -extended function over $X^{\prime}$ of $f$ is a multifunction $F: X^{\prime} \rightarrow Y$ such that

$$
\begin{aligned}
& \forall x \in X: F(x)=\{f(x)\}, \\
& \forall x \in X^{\prime} \backslash X: F(x) \in Z .
\end{aligned}
$$


Note 1 When we do not care about $Z$ in Definition 5, we just call $F$ an extended function over $X^{\prime}$ of $f$.

Notation 2 For simplicity, in Definition 5, for all $x \in X$ we write $F(x)=f(x)$ when no confusion can arise.

A $Z$-extended function $F$, as defined in Definition 5, corresponds to a function $g: X^{\prime} \rightarrow$ $Y \cup Z$ defined as

$$
g(x) \equiv \begin{cases}f(x) & \text { if } x \in X, \\ F(x) & \text { otherwise. }\end{cases}
$$

If $X^{\prime}=X$, then $f(x)=g(x)$ for all $x$ in $X$. By Definition 5, it is easy to prove the following theorem.

Theorem 2 Let $f, F$ and other notations be as in Definition 5. Then, for every subset $S$ of $X^{\prime}$, we have

$$
f(S) \equiv\{f(x) \mid x \in S \cap X\} \subseteq F(S) .
$$

Consider the case $X=D \subseteq \mathbb{R}^{n}, Y=\mathbb{R}^{m}$. It is easy to see that, for any function $f: D \subseteq \mathbb{R}^{n} \rightarrow \mathbb{R}^{m}$, there is only one $Z$-extended function from $\mathbb{R}^{n}$ to $\mathbb{R}^{m}$ if $Z$ has only one element, for example, when $Z$ is either $\{\emptyset\}$ or $\{\mathbb{R}\}$.

Example 3 The domain of the standard division $x \div y$ is $D_{\div}=\left\{(x, y) \in \mathbb{R}^{2} \mid y \neq 0\right\}$. The unique $\{\emptyset\}$-extended function over $\mathbb{R}$ of the standard division is defined as

$$
\div \varnothing(x, y) \equiv x \div \varnothing y \equiv \begin{cases}x / y & \text { if } y \neq 0 \\ \varnothing & \text { otherwise }\end{cases}
$$

The unique $\{\mathbb{R}\}$-extended function over $\mathbb{R}$ of the standard division is defined as

$$
\div \mathbb{R}(x, y) \equiv x \div \mathbb{R} y \equiv \begin{cases}x / y & \text { if } y \neq 0, \\ \mathbb{R} & \text { otherwise }\end{cases}
$$

The following is a $\{\emptyset, \mathbb{R}\}$-extended function over $\mathbb{R}$ of the standard division:

$$
\check{\star}_{\star}(x, y) \equiv x \div_{\star} y \equiv \begin{cases}x / y & \text { if } y \neq 0 \\ \emptyset & \text { if } x \neq 0, y=0 \\ \mathbb{R} & \text { otherwise }\end{cases}
$$

Example 4 The domain of the standard square root $\sqrt{x}$ is the interval $[0,+\infty]$. The unique $\{\emptyset\}$-extended function over $\mathbb{R}$ of the square root is defined as

$$
\sqrt{x} \emptyset \equiv \begin{array}{ll}
\sqrt{x} & \text { if } x \geq 0 \\
\varnothing & \text { otherwise }
\end{array}
$$

The unique $\{\mathbb{R}\}$-extended function over $\mathbb{R}$ of the square root is defined as

$$
\sqrt{x}^{\mathbb{R}} \equiv \begin{cases}\sqrt{x} & \text { if } x \geq 0 \\ \mathbb{R} & \text { otherwise }\end{cases}
$$


3.2 Extending interval forms

We now define the concept of interval form for a multifunction. This definition also holds for extended functions which are special cases of multifunctions.

Definition 6 Let $F: D \subseteq \mathbb{R}^{n} \rightarrow \mathbb{R}^{m}$ be a multifunction. A function $[F]: \mathbb{I}^{n} \rightarrow \mathbb{I}^{m}$ is called an interval form of $F$ if the following inclusion property holds:

$$
\forall x \in D, \forall \mathbf{x} \in \mathbb{I}^{n}: x \in \mathbf{x} \Rightarrow F(x) \subseteq[F](\mathbf{x}) .
$$

The natural interval form of $f$ is an instance of an interval form. The following theorem states the inclusion property of interval forms of multifunctions.

Theorem 3 Let $f: D \subseteq \mathbb{R}^{n} \rightarrow \mathbb{R}^{m}$ be a function and $F: D^{\prime} \supseteq D \rightarrow \mathbb{R}^{m}$ an extended function over $D^{\prime}$ of $f$. Then every interval form of $F$ is also an interval form of $f$.

Proof Let $[F]: \mathbb{I}^{n} \rightarrow \mathbb{I}^{m}$ be an interval form of $F$. Then for every $x \in D$ and every box $\mathbf{x} \in \mathbb{I}^{n}$ containing $x$, we have $f(x) \in\{f(x) \mid x \in \mathbf{x}\}=F(x) \subseteq[F](\mathbf{x})$.

Definition 7 (Interval Division: $\left[\div_{\emptyset}\right],[\div \mathbb{R}],\left[\div_{\star}\right]$ ) Let $\mathbf{x}=[\underline{x}, \bar{x}]$ and $\mathbf{y}=[y, \bar{y}]$ be two intervals. We define three natural interval forms of the division given by $(9, \overline{10})$ and $(11)$, respectively:

$$
\begin{aligned}
& {[\div \varnothing](\mathbf{x}, \mathbf{y}) \equiv \mathbf{x}\left[\div_{\varnothing}\right] \mathbf{y} \equiv \begin{cases}\emptyset & \text { if } \mathbf{y}=[0,0], \\
{[0,0]} & \text { else if } \mathbf{x}=[0,0], \\
\mathbf{x} \div \mathbf{y} & \text { else if } 0 \notin \mathbf{y}, \\
{[\underline{x} / \bar{y},+\infty]} & \text { else if } \underline{x} \geq 0 \wedge y=0, \\
{[-\infty, \underline{x} / y]} & \text { else if } \underline{x} \geq 0 \wedge \overline{\bar{y}}=0, \\
{[-\infty, \bar{x} / \bar{y}]} & \text { else if } \bar{x} \leq 0 \wedge y=0, \\
{[\bar{x} / \underline{y},+\infty]} & \text { else if } \bar{x} \leq 0 \wedge \bar{y}=0, \\
{[-\infty,+\infty]} & \text { otherwise; }\end{cases} } \\
& {[\div \mathbb{R}](\mathbf{x}, \mathbf{y}) \equiv \mathbf{x}[\div \mathbb{R}] \mathbf{y} \equiv \begin{cases}\mathbf{x} \div \mathbf{y} & \text { if } 0 \notin \mathbf{y}, \\
{[-\infty,+\infty]} & \text { otherwise }\end{cases} } \\
& {[\div \star \star x](\mathbf{x}, \mathbf{y}) \equiv \mathbf{x}\left[\div_{\star}\right] \mathbf{y} \equiv \begin{cases}\mathbf{x}[\div \varnothing] \mathbf{y} & \text { if } 0 \notin \mathbf{x} \vee 0 \notin \mathbf{y} \\
{[-\infty,+\infty]} & \text { otherwise. }\end{cases} }
\end{aligned}
$$

Some authors [8] use the tightest range of the division of two intervals; however, the result is not always an interval in that case.

Theorem 4 For any two intervals $\mathbf{x}$ and $\mathbf{y}$ in $\mathbb{I}$, we have

$$
\mathbf{x}[\div \emptyset] \mathbf{y} \subseteq \mathbf{x}\left[\leftarrow_{\star}\right] \mathbf{y} \subseteq \mathbf{x}[\div \mathbb{R}] \mathbf{y}
$$

Proof This is obvious from Definition 7.

Theorem 5 Let $x, y$, and $z$ be three real numbers living in three intervals $\mathbf{x}, \mathbf{y}$, and $\mathbf{z}$ in $\mathbb{I}$, respectively. Then we have

$$
\begin{aligned}
x=y * z & \Rightarrow z \in \mathbf{x} \diamond \mathbf{y} \text { for all } \diamond \in\{[\div \star x],[\div \mathbb{R}]\}, \\
z=x / y & \Rightarrow z \in \mathbf{x} \diamond \mathbf{y} \text { for all } \diamond \in\{[\div \emptyset],[\div \star],[\div \mathbb{R}]\} .
\end{aligned}
$$

Proof Notice that from a given equality $x=y * z$ we can deduce the following: 
- If $y \neq 0$, then $z=x / y$;

- If $y=0$, then $x=0$, and $z$ can take an arbitrary value.

The rest of the proof follows directly from Definition 7 .

On one hand, Theorem 5 shows that, when given the relation $x=y * z$, it is safe to use the domain reduction $\mathbf{z}:=\mathbf{x} \diamond \mathbf{y}$ for any $\diamond \in\left\{\left[\div \star{ }_{\star}\right],[\div \mathbb{R}]\right\}$. On the other hand, if the equality $z=x / y$ is given, we can safely reduce the domain of $z$ by the rule $\mathbf{z}:=\mathbf{x} \diamond \mathbf{y}$ for any $\diamond \in\left\{[\div \mathbb{R}],\left[\div \star{ }_{\star}\right],[\div \varnothing]\right\}$, because the case $y=0$ is not admitted by definition.

Theorem 4 and 5 show that the tightness of a natural interval form of a function defined on a subset of $\mathbb{R}^{n}$ usually depends on the underlying extended function. In turn, the extended function should be chosen based on the context of the computation. Many interval implementations (e.g., [30]) use the division $[\div \mathbb{R}]$ in all computations. However, from Theorem 5 we can see that it is safe to use the division $[\div \varnothing]$ in computations such as forward evaluations and use the division $\left[\leftarrow_{\star}\right]$ in computations such as backward propagations, as described in Sects. 2.1.2 and 4 .

\section{Forward-Backward propagation on DAG representations}

In [21] the authors have adapted to DAGs the forward evaluations and backward propagations defined on trees [5]. The forward and backward procedures they propose work at the graph level, which means that all the nodes of the graph are forward evaluated then backward propagated at once.

In this section we shift the original definitions to the node level. The goal is to make it possible to run forward evaluation and backward propagation adaptively on particular nodes only. As shown in Sect. 5, the nodes will be chosen depending on their ability to cause changes in the domain ranges of their related expressions. This section also introduces the notion of a partial DAG representation which will make it possible to perform branch-and-prune search without creating multiple DAGs. This section uses Notation 1.

\subsection{Forward evaluation on DAG representations}

Forward evaluation at a node $\mathbf{N}$ is concerned with evaluating the range of the expression represented by $\mathbf{N}$ on the basis of the node ranges of the children of $\mathbf{N}$.

Consider the DAG representation of a factorable NCSP of the form (1). Let $\mathbf{N}$ be a node that is not the ground node and that has $k$ children: $\mathbf{C}_{1}, \ldots, \mathbf{C}_{k}$. Suppose the operation represented by $\mathbf{N}$ is a function $h: D_{h} \subseteq \mathbb{R}^{k} \rightarrow \mathbb{R}$. The relation between $\mathbf{N}$ and its children is given by $\vartheta_{\mathbf{N}}=h\left(\vartheta_{\mathbf{C}_{1}}, \ldots, \vartheta_{\mathbf{C}_{k}}\right)$. We define the forward evaluation at node $\mathbf{N}$ as follows.

Definition 8 (Forward Evaluation) Consider a node $\mathbf{N}$ and its operation $h$ as described above. Let $[h]$ be an interval form of the $\{\emptyset\}$-extended function over $\mathbb{R}$ of $h$. The forward evaluation at $\mathbf{N}$ using $[h]$ is defined and denoted by

$$
\mathrm{FE}(\mathbf{N},[h]) \equiv\left(\tau_{\mathbf{N}}:=\tau_{\mathbf{N}} \cap[h]\left(\tau_{\mathbf{C}_{1}}, \ldots, \tau_{\mathbf{C}_{k}}\right)\right) .
$$

Example 5 Consider the node $\mathbf{N}_{7}$ in Fig. $3, h(z) \equiv \sqrt{z}$, where $z \equiv \vartheta_{\mathbf{N}_{5}}$. We can use any interval form $[h]$ of the $\{\varnothing\}$-extended function over $\mathbb{R}$ of $h$, which is the function $\sqrt{z}^{\emptyset}$ defined by (12), for the forward evaluation in (20). We can use, for example, the natural interval form $\mathbf{h}(\mathbf{z}) \equiv \sqrt{\mathbf{z}}$ in place of $[h]$ in (20). 
Remark 2 We can also replace $[h]$ in (20) with an interval form of the recursive subexpression whose variables are the initial variables. For instance, we can replace the interval form $[h]$ of the node $\mathbf{N}_{7}$ in Fig. 3 with the natural interval form of the recursive subexpression $(\sqrt{x y})$ composed of the nodes $\mathbf{N}_{7}, \mathbf{N}_{4}, \mathbf{N}_{1}$, and $\mathbf{N}_{2}$. That is, we can replace [ $\left.h\right]$ with the bivariate interval function $\sqrt{\mathbf{x y}}$.

In our implementation, we use the natural interval form for simplicity. The natural interval form of the function $h\left(x_{1}, \ldots, x_{k}\right)=a_{0}+a_{1} x_{1}+\cdots+a_{k} x_{k}$ is the function $\mathbf{h}\left(\mathbf{x}_{1}, \ldots, \mathbf{x}_{k}\right)=$ $a_{0}+a_{1} \mathbf{x}_{1}+\cdots+a_{k} \mathbf{x}_{k} \cdot{ }^{1}$ Similarly, the natural interval form of the function $h\left(x_{1}, \ldots, x_{k}\right)=$ $a x_{1} \ldots x_{k}$ is the function $\mathbf{h}\left(\mathbf{x}_{1}, \ldots, \mathbf{x}_{k}\right)=a \mathbf{x}_{1} \ldots \mathbf{x}_{k}$. The division of two reals has multiple natural interval forms because it is not defined when the denominator is zero (see Sects. 3.1 and 3.2). In Definition 7, we have provided three versions that can be called the natural interval forms of the real division: $\left[\div_{\emptyset}\right],\left[\leftarrow_{\star}\right]$, and $[\div \mathbb{R}]$. They all can be used in the forward evaluation defined by (20) if $h$ is the real division.

Theorem 6 (Correctness) Consider the DAG representation of a factorable numerical CSP given in (1). The forward evaluation defined in Definition 8, when applied to any node, never discards a solution of the considered problem.

Proof For every solution of the considered problem, there exists an assignment of values from the intervals $\tau_{\mathbf{N}}, \tau_{\mathbf{C}_{1}}, \ldots, \tau_{\mathbf{C}_{k}}$ to the variables $\vartheta_{\mathbf{N}}, \vartheta_{\mathbf{C}_{1}}, \ldots, \vartheta_{\mathbf{C}_{k}}$, respectively, such that $\vartheta_{\mathbf{N}}=h\left(\vartheta_{\mathbf{C}_{1}}, \ldots, \vartheta_{\mathbf{C}_{k}}\right)$. Because $[h]$ is an interval form of the $\{\emptyset\}$-extended function over $\mathbb{R}$ of $h$, it follows from Theorem 3 that $h\left(\vartheta_{\mathbf{C}_{1}}, \ldots, \vartheta_{\mathbf{C}_{k}}\right) \in[h]\left(\tau_{\mathbf{C}_{1}}, \ldots, \tau_{\mathbf{C}_{k}}\right)$. Thus, $\vartheta_{\mathbf{N}} \in \tau_{\mathbf{N}} \cap[h]\left(\tau_{\mathbf{C}_{1}}, \ldots, \tau_{\mathbf{C}_{k}}\right)$. The proof is, therefore, complete.

\subsection{Backward propagation on DAG representations}

Backward propagation at a node $\mathbf{N}$ will reduce the node range of each child of $\mathbf{N}$ on the basis of the node ranges of $\mathbf{N}$ and on the node ranges of its other children.

Consider the DAG representation of a factorable NCSP of the form (1). Let $\mathbf{N}$ be a node that is not the ground node and that has $k$ children: $\mathbf{C}_{1}, \ldots, \mathbf{C}_{k}$. The operation represented by $\mathbf{N}$ is a function $h: D_{h} \subseteq \mathbb{R}^{k} \rightarrow \mathbb{R}$. The backward propagation attempts to prune each node range $\tau_{\mathbf{C}_{i}}$ of $\mathbf{C}_{i}$ based on the node range $\tau_{\mathbf{N}}$ of $\mathbf{N}$ and based on the node ranges of the other children, where $1 \leq i \leq k$. In other words, for each child $\mathbf{C}_{i}$, the backward propagation attempts to encloses the $i$ th projection of the relation $\vartheta_{\mathbf{N}}=h\left(\vartheta_{\mathbf{C}_{1}}, \ldots, \vartheta_{\mathbf{C}_{k}}\right)$ on the variable $\vartheta_{\mathbf{C}_{i}}$ in a tight interval. This procedure is called the $i$ th backward propagation at $\mathbf{N}$ and denoted by $\operatorname{BP}\left(\mathbf{N}, \mathbf{C}_{i}\right)$. We define the following as the backward propagation at $\mathbf{N}$ :

$$
\mathrm{BP}(\mathbf{N}) \equiv\left\{\mathrm{BP}\left(\mathbf{N}, \mathbf{C}_{1}\right), \ldots, \mathrm{BP}\left(\mathbf{N}, \mathbf{C}_{k}\right)\right\} .
$$

Although the exact projection of a relation is expensive, in general, an enclosure of the exact projection of an elementary operation can often be obtained at low cost. Indeed, suppose that we can infer from the relation $\vartheta_{\mathbf{N}}=h\left(\vartheta_{\mathbf{C}_{1}}, \ldots, \vartheta_{\mathbf{C}_{k}}\right)$ an equivalent relation

$$
\vartheta_{\mathbf{C}_{i}}=g_{i}\left(\vartheta_{\mathbf{N}}, \vartheta_{\mathbf{C}_{1}}, \ldots, \vartheta_{\mathbf{C}_{i-1}}, \vartheta_{\mathbf{C}_{i+1}}, \ldots, \vartheta_{\mathbf{C}_{k}}\right)
$$

for some $i \in\{1, \ldots, k\}$, where $g_{i}$ is a function from $D_{g} \subseteq \mathbb{R}^{k}$ to $\mathbb{R}$ such that

$$
D_{g} \supseteq \tau_{\mathbf{N}} \times \tau_{\mathbf{C}_{1}} \times \cdots \times \tau_{\mathbf{C}_{i-1}} \times \tau_{\mathbf{C}_{i+1}} \times \cdots \times \tau_{\mathbf{C}_{k}} .
$$

\footnotetext{
1 Note that if the coefficients $a_{0}, \ldots, a_{k}$ are real and we are working on the floating-point number system, we can replace each $a_{i}$ in $\mathbf{h}$ with the smallest interval containing it, where $1 \leq i \leq k$.
} 
Let $\left[g_{i}\right]$ be an interval form of the $\{\varnothing\}$-extended function over $\mathbb{R}$ of $g_{i}$. The $i$ th backward propagation, denoted $\mathrm{BP}\left(\mathbf{N}, \mathbf{C}_{i}\right)$, can then be defined as

$$
\operatorname{BP}\left(\mathbf{N}, \mathbf{C}_{i}\right) \equiv\left(\tau_{\mathbf{C}_{i}}:=\tau_{\mathbf{C}_{i}} \cap\left[g_{i}\right]\left(\tau_{\mathbf{N}}, \tau_{\mathbf{C}_{1}}, \ldots, \tau_{\mathbf{C}_{i-1}}, \tau_{\mathbf{C}_{i+1}}, \ldots, \tau_{\mathbf{C}_{k}}\right)\right) .
$$

In case we cannot infer such a function $g_{i}$, more complicated rules have to be constructed in order to obtain the $i$ th projection of the relation $\mathbf{N}=f\left(\mathbf{C}_{1}, \ldots, \mathbf{C}_{k}\right)$ if the cost is low, otherwise the relation can be ignored. Fortunately, we can tightly enclose such projections at low cost for most elementary operations, as shown in Definition 9.

Remark 3 In general, the relation $x=y * z$ and the relation $z=x / y$ are not equivalent because the latter discards the case $y=0$ while the former does not.

Example 6 Consider the node $\mathbf{N}_{10}$ in Fig. 3. The relation given at $\mathbf{N}_{10}$ is $\vartheta_{\mathbf{N}_{10}}=h\left(\vartheta_{\mathbf{N}_{5}}, \vartheta_{\mathbf{N}_{6}}\right.$, $\left.\vartheta_{\mathbf{N}_{8}}\right)$, where the function $h$ is defined as $h\left(x_{1}, x_{2}, x_{3}\right) \equiv-2 x_{1}+3 x_{2}+x_{3}$. Therefore, we can infer three equivalent relations:

$$
\begin{aligned}
& \vartheta_{\mathbf{N}_{5}}=g_{1}\left(\vartheta_{\mathbf{N}_{10}}, \vartheta_{\mathbf{N}_{6}}, \vartheta_{\mathbf{N}_{8}}\right), \\
& \vartheta_{\mathbf{N}_{6}}=g_{2}\left(\vartheta_{\mathbf{N}_{10}}, \vartheta_{\mathbf{N}_{5}}, \vartheta_{\mathbf{N}_{8}}\right), \\
& \vartheta_{\mathbf{N}_{8}}=g_{3}\left(\vartheta_{\mathbf{N}_{10}}, \vartheta_{\mathbf{N}_{5}}, \vartheta_{\mathbf{N}_{6}}\right),
\end{aligned}
$$

where the three functions $g_{1}, g_{2}$ and $g_{3}$ are defined as follows:

$$
\begin{aligned}
& g_{1}\left(x_{1}, x_{2}, x_{3}\right) \equiv\left(-x_{1}+3 x_{2}+x_{3}\right) / 2, \\
& g_{2}\left(x_{1}, x_{2}, x_{3}\right) \equiv\left(x_{1}+2 x_{2}-x_{3}\right) / 3, \\
& g_{3}\left(x_{1}, x_{2}, x_{3}\right) \equiv x_{1}+2 x_{2}-3 x_{3} .
\end{aligned}
$$

Definition 9 (Backward Propagation Rule) Let $h$ be the elementary operation represented by node $\mathbf{N}$, as discussed above. We use the notation $\oslash$ to mean that either the division $\left[\leftarrow_{\star}\right]$ or the division $[\div \mathbb{R}]$ can be used at the place the notation $\oslash$ appears, but the former is better. The rules for backward propagation are given as follows:

1. If $h$ is a univariate function such as sqr, sqrt, exp, and log and if $[h]$ is an interval form of the $\{\emptyset\}$-extended function of $h$, we define

$$
\operatorname{BP}\left(\mathbf{N}, \mathbf{C}_{1}\right) \equiv\left(\tau_{\mathbf{C}_{1}}:=\tau_{\mathbf{C}_{1}} \cap\left[h^{-1}\right]\left(\tau_{\mathbf{N}}\right)\right),
$$

where the notation of interval form, $\left[h^{-1}\right](\mathbf{x})$, shall denote the union of some intervals that contains the inverse image $h^{-1}(\mathbf{x})$;

2. If $h$ is defined as $h\left(x_{1}, \ldots, x_{k}\right) \equiv a_{0}+a_{1} x_{1}+\cdots+a_{k} x_{k}$, we define for $i=1, \ldots, k$ :

$$
\mathrm{BP}\left(\mathbf{N}, \mathbf{C}_{i}\right) \equiv\left(\tau_{\mathbf{C}_{i}}:=\tau_{\mathbf{C}_{i}} \cap\left(\left(\tau_{\mathbf{N}}-a_{0}-\sum_{j=1 ; j \neq i}^{k} a_{j} * \tau_{\mathbf{C}_{j}}\right) \oslash a_{i}\right)\right) ;
$$

3. If $h$ is defined as $h\left(x_{1}, \ldots, x_{k}\right) \equiv a x_{1} \ldots x_{k}$, we define for $i=1, \ldots, k$ :

$$
\mathrm{BP}\left(\mathbf{N}, \mathbf{C}_{i}\right) \equiv\left(\tau_{\mathbf{C}_{i}}:=\tau_{\mathbf{C}_{i}} \cap\left(\tau_{\mathbf{N}} \oslash\left(a * \prod_{j=1 ; j \neq i}^{k} \tau_{\mathbf{C}_{j}}\right)\right) ;\right.
$$

4. If $h$ is defined as $h(x, y) \equiv x / y$, we define

$$
\begin{aligned}
& \mathrm{BP}\left(\mathbf{N}, \mathbf{C}_{1}\right) \equiv\left(\tau_{\mathbf{C}_{1}}:=\tau_{\mathbf{C}_{1}} \cap\left(\tau_{\mathbf{N}} * \tau_{\mathbf{C}_{2}}\right)\right), \\
& \operatorname{BP}\left(\mathbf{N}, \mathbf{C}_{2}\right) \equiv\left(\tau_{\mathbf{C}_{2}}:=\tau_{\mathbf{C}_{2}} \cap\left(\tau_{\mathbf{C}_{1}} \oslash \tau_{\mathbf{N}}\right)\right) .
\end{aligned}
$$


The following theorem states the correctness of the backward propagation rules given in Definition 9.

Theorem 7 (Correctness) Consider the DAG representation of a factorable numerical CSP given in (1). The backward propagation defined in Definition 9, when applied to any node, never discards any solution of the considered problem.

Proof By an argument similar to the Proof of Theorem 6, we have that the result for the first rule is due to the definition of $h^{-1}$ in Definition 4, and for the other rules, to Theorem 5 and to the inclusion property of the operations,+- , and $*$ in (standard) interval arithmetic.

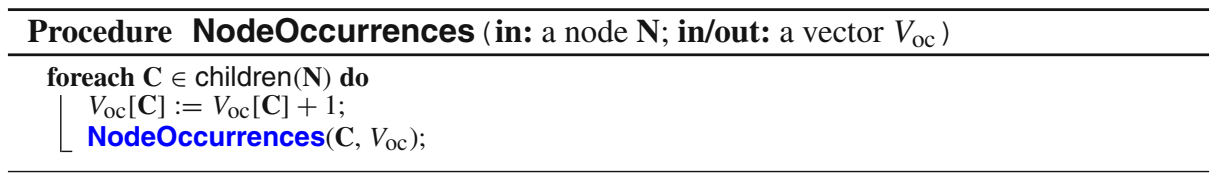

\subsection{Partial DAG representations}

When solving NCSPs using a branch-and-prune scheme, the branching step splits the problem into subproblems, potentially easier to solve. Each subproblem often consists of the following two components:

1. a subset of the initial constraints set, called the set of running constraints;

2. a sequence of subdomains for the involved variables.

If we use DAG representations in the pruning steps, we have to construct a DAG representation for each subproblem. A simple way is to construct a new DAG explicitly to represent each subproblem. However, the total cost of creating such DAGs for the whole solving process is potentially high, because there are often a huge number of branching steps during the solution process.

Alternatively, we propose to attach a piece of restriction information to the DAG representation of the initial problem so that it can be interpreted as the DAG representation of a subproblem (without creating a new DAG). When using such pieces of restriction information, it is possible to perform forward evaluations and backward propagations on the DAG representation of the initial problem without increasing the time and space for dealing with DAGs. A combination of such a piece of restriction information and the DAG representation of the initial problem is called the partial DAG representation of a subproblem. It is also called, for convenience, a partial DAG representation of the initial problem. For example, partial DAG representations of the problem (2) are depicted in Fig. 4. We use partial DAG representations instead of DAG representations in our new propagation algorithm (Sect. 5).

In order to represent a subproblem with a set of running constraints without having to create a new DAG, we use a vector $V_{\mathrm{oc}}$ whose size equals the number of nodes in the DAG representation $D_{\mathbf{G}}$ of the initial problem. For each node $\mathbf{N}$ of $D_{\mathbf{G}}$, we use the entry $V_{\text {oc }}[\mathbf{N}]$ to count the number of occurrences of $\mathbf{N}$ in the recursive composition of the running constraints. We present a simple recursive procedure, called NodeOccurrences, to compute such a vector.

If we invoke NodeOccurrences at all the nodes representing the running constraints, then each entry $V_{\mathrm{oc}}[\mathbf{N}]$ will contains the number of occurrences of $\mathbf{N}$ in the recursive composition of the running constraints. In particular, we have $V_{\mathrm{oc}}[\mathbf{N}]=0$ if and only if $\mathbf{N}$ is not in 


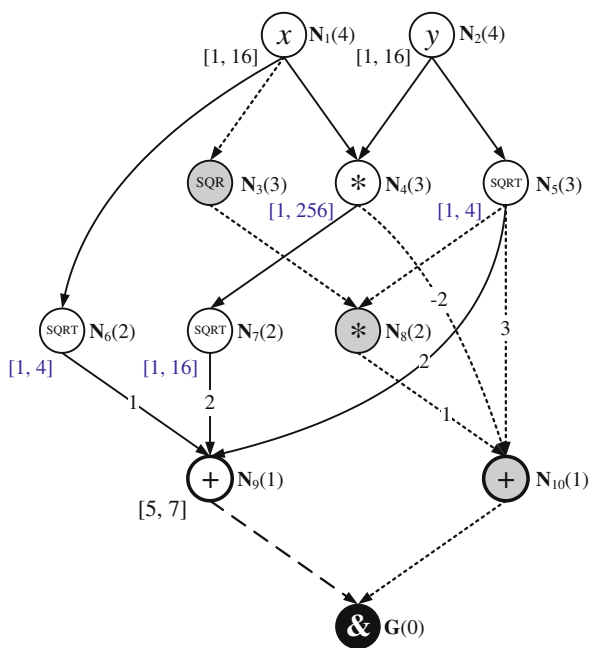

(a)

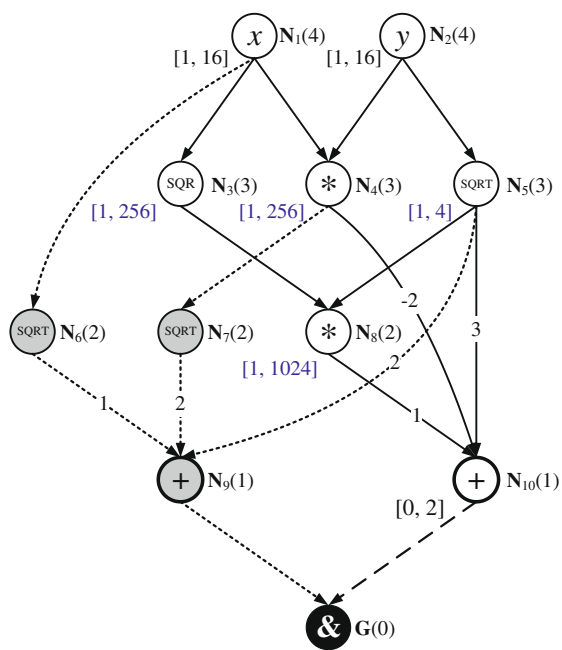

(b)

Fig. 4 The partial DAG representations of the problem (2) in the cases: (a) the first constraint is the unique running constraint; and (b) the second constraint is the unique running constraint. The grey nodes and dotted edges are ignored. The node levels are given in parenthesis

the representation of the running constraints. Therefore, by combining $D_{\mathbf{G}}$ with a vector $V_{\mathrm{oc}}$, we have the so-called partial DAG representation for a subproblem. In computations, we can use partial DAG representations in a way similar to the way we use DAG representations, except that we ignore every node $\mathbf{N}$ corresponding to $V_{\mathrm{oc}}[\mathbf{N}]=0$.

\section{Constraint propagation on partial DAG representations}

This section presents our new algorithm, called FBPD which generalizes to DAGs the HC4 algorithm originally proposed in [5] for tree representations of constraints.

We recall that, at each iteration, the $\mathbf{H C 4}$ algorithm invokes the HC4revise algorithm (see Sect. 2.1.2), which in turn consists of two recursive propagation procedures: a recursive forward evaluation (RFE) and a recursive backward propagation (RBP). In order to reduce the node ranges and, in particular, the domains of variables, RFE performs forward evaluations at all nodes of the tree representation of a constraint in the post-order and then RBP performs backward propagations at all nodes of this tree representation in the pre-order.

Consider a factorable NCSP. We propose in this section a new propagation algorithm that enhances the $\mathbf{H C 4}$ algorithm by:

- working on (partial) DAG representations, instead of tree representations, of the considered problem;

- exploiting the common subexpressions of the constraints as the influence of the constraints on each other;

- flexibly choosing nodes at which the forward evaluations and backward propagations are performed.

Moreover, the nature of the new propagation algorithm makes it possible to use different interval forms at different steps of the propagation. As discussed above, the new propagation 


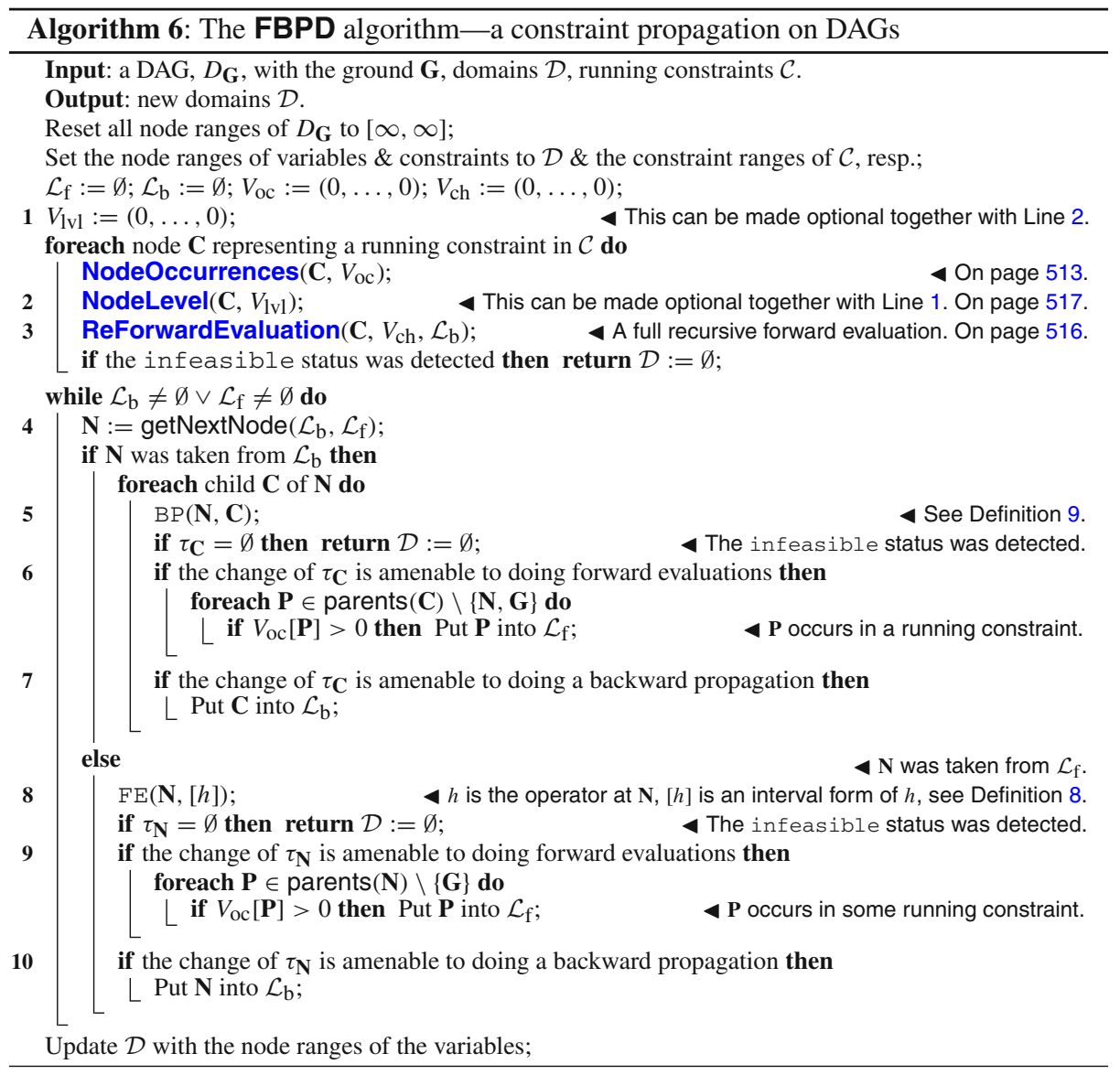

algorithm works on partial DAG representations of the initial problem to reduce time and space when dealing with DAG representations. Since the main processes of the new algorithm are forward evaluations and backward propagations, we call it the Forward-Backward Propagation on DAGs (FBPD). The main steps of the FBPD algorithm are presented in Algorithm 6.

The FBPD algorithm takes as input a subproblem that is represented by the DAG representation $D_{\mathbf{G}}$ of the initial problem, a sequence $\mathcal{D}$ of subdomains of variables, and a set $\mathcal{C}$ of running constraints of the subproblem. Like the HC4 algorithm, the FBPD algorithm relies on two types of processes: forward evaluation and backward propagation. Unlike the HC4 algorithm, the FBPD algorithm, however, performs these processes on the basis of one node at a time rather than all nodes at once. The choice of the next node for the next process in the FBPD algorithm is adaptively made based on the results of the previous processes. Moreover, in the FBPD algorithm the choice of the interval form $[h]$ of an operation $h$ for forward evaluations and backward propagations is not necessarily fixed. The interval form [h] can be chosen statically or dynamically based on the nature of $h$ at the current context.

In the next subsections, we describe in detail the procedures that are not made explicit in Algorithm 6. 


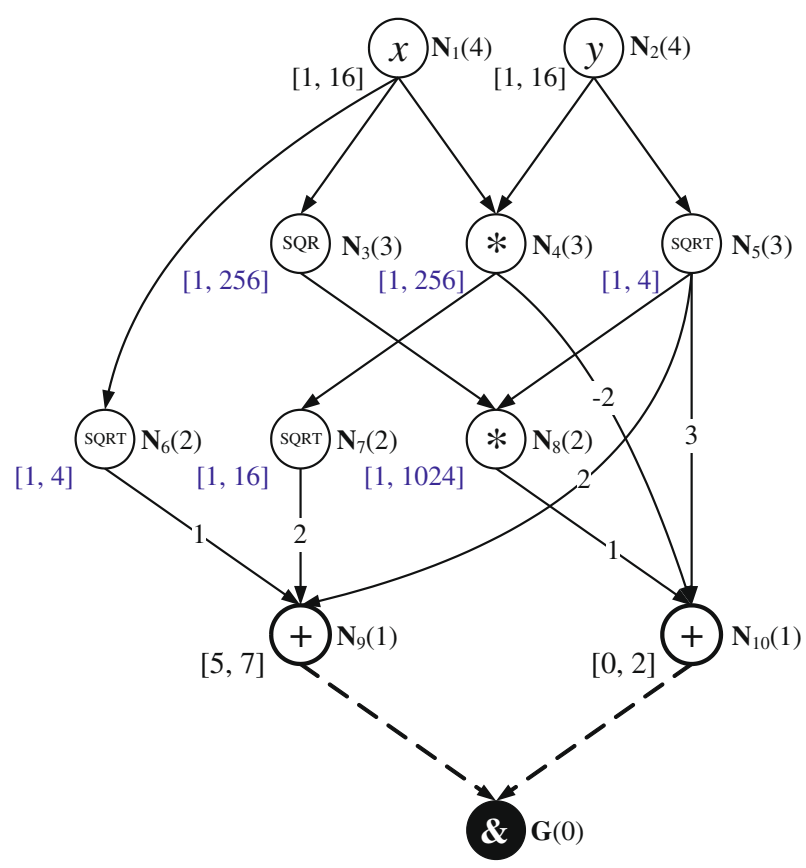

Fig. 5 The DAG representation of the system (2) after a recursive forward evaluation

\subsection{Initialization phase}

Similarly to the HC4 algorithm, the FBPD algorithm performs a recursive forward evaluation at the initialization phase (Line 3 in Algorithm 6) to evaluate the node ranges of all nodes in the partial DAG representation of the subproblem. That is, FBPD computes the node ranges of the nodes of $D_{\mathbf{G}}$ that correspond to nonzero entries in $V_{\text {oc }}$. Procedure ReForwardEvaluation provides such an algorithm. In order to avoid evaluating the same subexpressions multiple times, we use a vector, $V_{\mathrm{ch}}$, to mark the caching status of node ranges. A node $\mathbf{N}$ is marked as "cached" by setting $V_{\mathrm{ch}}[\mathbf{N}]:=1$ if its node range has already been computed.

The result of the recursive forward evaluation of the NCSP given in (2) is depicted in Fig. 4 (in case only one constraint is running in the subproblem) and Fig. 5 (in case both constraints are running in the subproblem).

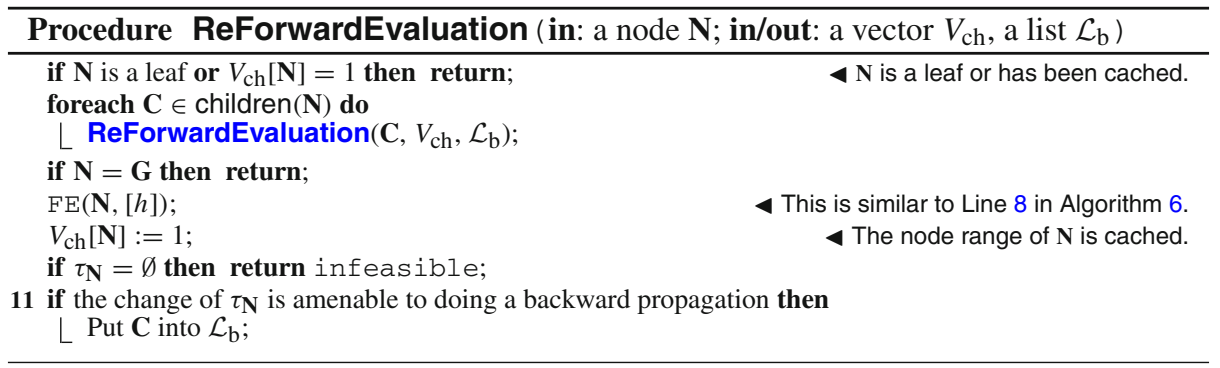




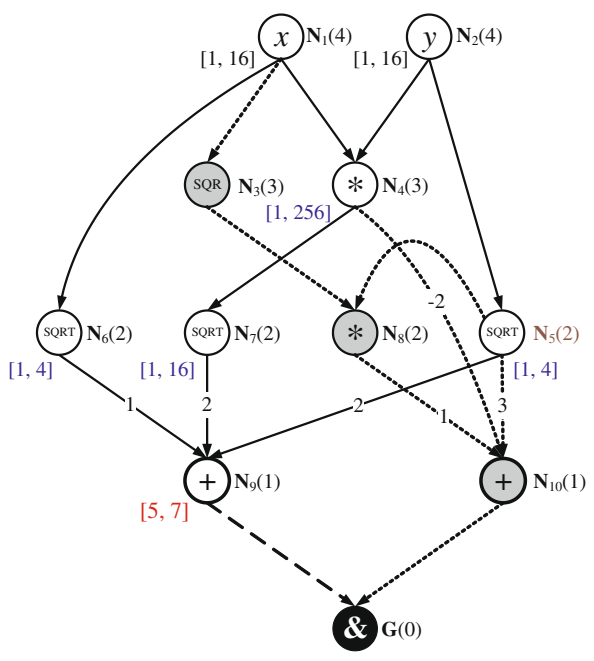

(a)

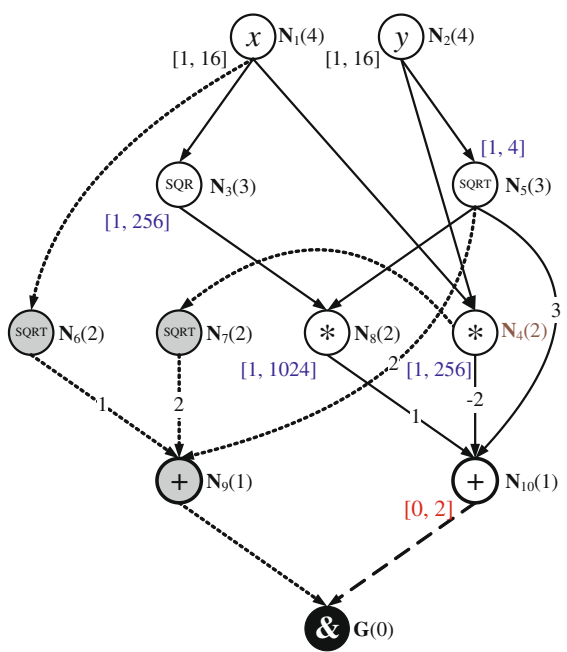

(b)

Fig. 6 The node levels are updated at each call to the FBPD algorithm

\subsection{Getting the next node}

The FBPD algorithm uses two waiting lists, $\mathcal{L}_{\mathrm{f}}$ and $\mathcal{L}_{\mathrm{b}}$, to store the nodes waiting for further processing. The first list, $\mathcal{L}_{\mathrm{f}}$, is a list of nodes that is scheduled for forward evaluation, that is, for evaluating its node range based on the node ranges of its children. The second list, $\mathcal{L}_{\mathrm{b}}$, is a list of nodes waiting for backward evaluation (reducing the node ranges of their children based on their own node ranges). In general, the nodes in $\mathcal{L}_{\mathrm{f}}$ should be sorted such that the forward evaluation at a node is performed after the forward evaluations at its children. Analogously, the nodes in $\mathcal{L}_{\mathrm{b}}$ should be sorted such that the backward propagation at a node is performed before the backward propagations at its children.

Procedure NodeLevel assigns to each node a node level such that the node level of an arbitrary node is smaller than the node levels of its descendants (see Theorem 1). We then sort the nodes of $\mathcal{L}_{\mathrm{b}}$ and $\mathcal{L}_{\mathrm{f}}$ in ascending order and descending order of node levels, respectively, to meet the above requirements.

The call to Procedure NodeLevel at Line 2 in Algorithm 6 can be made optional as follows. The first option is to invoke NodeLevel only at the first call to the FBPD algorithm. The node levels of the initial DAG still meet the requirements on the ordering of the waiting lists. The numbers in brackets following the node names in Fig. 4 are the node levels computed for the initial DAG representation. Figure 6 illustrates the second option that is to invoke NodeLevel at Line 2 in Algorithm 6 each time the FBPD algorithm is invoked.

The getNextNode function at Line 4 in Algorithm 6 chooses one of the two nodes at the beginning of $\mathcal{L}_{\mathrm{b}}$ and $\mathcal{L}_{\mathrm{f}}$. The choosing strategy we use in our implementation is backward

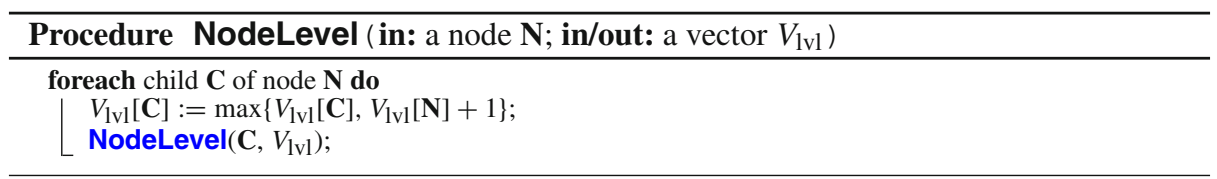


propagation first, that is, taking the node at the beginning of $\mathcal{L}_{\mathrm{b}}$ whenever $\mathcal{L}_{\mathrm{b}}$ is not empty. Of course, more involved strategies can also be considered.

5.3 Is the change of a node range amenable to further processing?

For simplicity, at the Lines 6, 7, 9 and 10 of Algorithm 6 we have only briefly presented the procedures to check if the change of a node range is amenable to a forward evaluation or backward propagation. We now describe them in detail.

Let $\mathbf{M}$ denote the node $\mathbf{C}$ at Line 5 or the node $\mathbf{N}$ at Line 8 in Algorithm 6. The backward propagation at Line 5 and the forward evaluation at Line 8 in Algorithm 6 have the same form

$$
\tau_{\mathbf{M}}:=\tau_{\mathbf{M}} \cap \mathbf{y},
$$

where $\mathbf{y}$ is the interval computed by the forward evaluation or backward propagation right before intersecting with $\tau_{\mathbf{M}}$ at the considered line. Let $W_{\text {old }}$ and $W_{\text {new }}$ be the widths of $\tau_{\mathbf{M}}$ and $\tau_{\mathbf{M}} \cap \mathbf{y}$, respectively, right before the intersection.

In practice, the change of $\tau_{\mathbf{M}}$ after performing (23) is amenable to doing forward evaluations at M's parents if both conditions $W_{\text {new }}<r_{\mathrm{f}} * W_{\text {old }}$ and $W_{\text {new }}+d_{\mathrm{f}}<W_{\text {old }}$ hold, where $r_{\mathrm{f}} \in(0,1]$ and $d_{\mathrm{f}} \geq 0$ are real parameters.

Similarly, the change of $\tau_{\mathbf{M}}$ after performing the intersection (23) is amenable to doing a backward propagation at $\mathbf{M}$ if both conditions $W_{\text {new }}<r_{\mathrm{b}} * W_{\text {old }}$ and $W_{\text {new }}+d_{\mathrm{b}}<W_{\text {old }}$ hold, where $r_{\mathrm{b}} \in(0,1]$ and $d_{\mathrm{b}} \geq 0$ are real parameters. In addition to that, the condition $\mathbf{y} \nsubseteq \tau_{\mathbf{M}}$ must also hold if $\mathbf{y}$ has been computed by the forward evaluation (at Line 8).

The parameters $r_{\mathrm{f}}, d_{\mathrm{f}}, r_{\mathrm{b}}$ and $d_{\mathrm{b}}$ can be predetermined or dynamically computed. In our implementation these parameters are predetermined.

\subsection{Properties of the new propagation algorithm}

The FBPD algorithm is contractive and correct in the following sense.

Theorem 8 Let $\Phi: \mathbb{I}^{n} \rightarrow \mathbb{I}^{n}$ be a function representing the FBPD algorithm. This function takes as input the domains of the input problem in the form of a box $\mathbf{x} \in \mathbb{I}^{n}$ and returns a box in $\mathbb{I}^{n}$, denoted as $\Phi(\mathbf{x})$, that represents the domains of the output problem of the FBPD algorithm. If the input problem contains only the operations $h$ defined in Definition 8 and 9, then the FBPD algorithm terminates at a finite number of iterations and the following properties hold:

$$
\begin{aligned}
& \text { (Contractiveness) } \Phi(\mathbf{x}) \subseteq \mathbf{x}, \\
& \text { (Correctness) } \Phi(\mathbf{x}) \supseteq \mathbf{x} \cap S,
\end{aligned}
$$

where $S$ is the exact solution set of the input problem.

Proof All the node ranges in the DAG representation of the considered problem are never inflated at each step of the FBPD algorithm. Hence, the FBPD algorithm must terminate at a finite number of iterations because of the finite nature of floating-point numbers. In particular, the ranges of the nodes representing the variables are never inflated. Thus, the property (24) holds. Moreover, the forward evaluations and backward propagations used in the FBPD algorithm are defined in Definition 8 and 9. It follows from Theorem 6 and 7 that they never discard a solution. Therefore, the property (25) also holds. 
Theorem 9 Let $\mathbf{M}$ be a node in the (partial) DAG representation of the output subproblem of the FBPD algorithm.

- Suppose that the FBPD algorithm uses a fixed interval form [ $h]$ of the elementary operation $h$ presented by $\mathbf{M}$ at all steps. Let $\mathbf{y}$ be the interval computed right before the last intersection in the forward evaluation $\mathrm{FE}(\mathbf{M},[h])$ (Definition 8). Then the following holds:

$$
\mathrm{w}\left(\tau_{\mathbf{M}} \cap \mathbf{y}\right) \geq r_{\mathrm{f}} * \mathrm{w}\left(\tau_{\mathbf{M}}\right) \vee \mathrm{w}\left(\tau_{\mathbf{M}} \cap \mathbf{y}\right)+d_{\mathrm{f}} \geq \mathrm{w}\left(\tau_{\mathbf{M}}\right) .
$$

- Suppose that $\mathbf{N}$ is a parent of $\mathbf{M}$ and that the FBPD algorithm uses fixed interval forms of elementary operations in $\mathrm{BP}(\mathbf{N}, \mathbf{M})$ at all steps. Let $\mathbf{z}$ be the interval computed right before the last intersection in the backward propagation $\mathrm{BP}(\mathbf{N}, \mathbf{M})$ (Definition 9). Then the following holds:

$$
\mathrm{w}\left(\tau_{\mathbf{M}} \cap \mathbf{z}\right) \geq r_{\mathrm{b}} * \mathrm{w}\left(\tau_{\mathbf{M}}\right) \vee \mathrm{w}\left(\tau_{\mathbf{M}} \cap \mathbf{z}\right)+d_{\mathrm{b}} \geq \mathrm{w}\left(\tau_{\mathbf{M}}\right) .
$$

Proof This follows directly from the discussion in Sect. 5.3.

\section{Coordinating constraint propagation and search}

Next, we consider the issue of coordinating constraint propagation and search for solving NCSPs in the branch-and-prune framework - the most common framework for exhaustively solving NCSPs. The most widely used search algorithm is based on the bisection of domains, and is hence called bisection search. It is suitable for solving problems with isolated solutions. However, it is often inefficient for solving problems with a continuum of solutions. For such problems, therefore, we need more advanced search techniques. We consider the issue of integrating the FBPD algorithm into a generic branch-and-prune search algorithm, called BnPSearch, described in Algorithm 9.

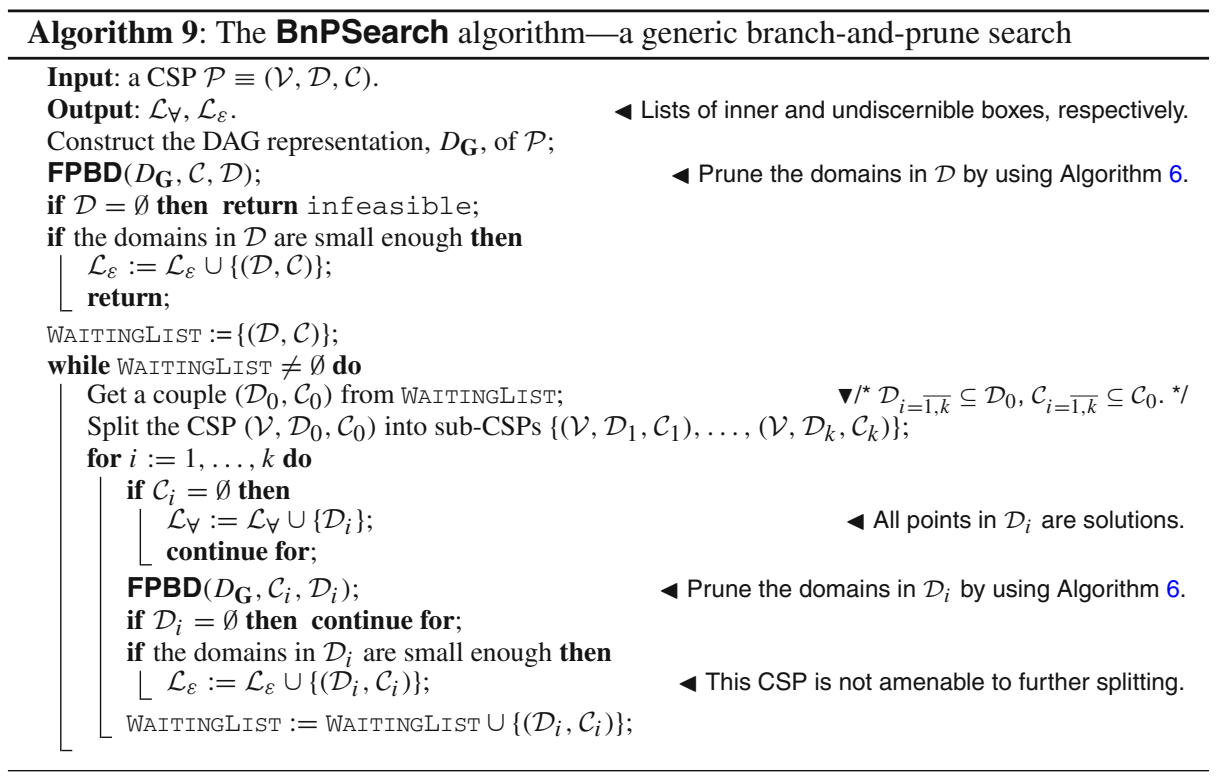


The BnPSearch algorithm produces two lists: $\mathcal{L}_{\forall}, \mathcal{L}_{\varepsilon}$. The first list, $\mathcal{L}_{\forall}$, consists of completely feasible domain boxes, called inner boxes or feasible boxes. That is, all points of a box in $\mathcal{L}_{\forall}$ are a solution of the problem. The second list, $\mathcal{L}_{\varepsilon}$, consists of subproblems, each consisting of a domain box and a set of running constraints. Each domain box of a subproblem in $\mathcal{L}_{\varepsilon}$ is canonical or smaller than the required precision $\varepsilon$. These domain boxes are called undiscernible boxes.

Owning to Theorem 8 and the finite nature of the floating-point number system, it is easy to prove that the branch-and-prune search in Algorithm 9 terminates after a finite number of iterations. Moreover, this search algorithm never discards any solution. Note that the UCA6 and $\mathbf{U C A 6}^{+}$algorithms in [22,25] are specific instances of this generic search.

\section{Experiments}

We have carried out experiments on the FBPD algorithm and two other well-known state-ofthe-art interval constraint propagation techniques. The first propagation technique is a variant of box consistency [4] implemented in a commercial product, ILOG Solver (v6.0), hereafter denoted as BOX. The second constraint propagation technique is the HC4 algorithm (see Sect. 2.1.2) in an implementation by IRIN based on the JAIL interval library and the arcchi platform. These two constraint propagation techniques have been used since they have been readily available and constitute the basic machinery behind efficient state-of-the-art methods for solving numerical CSP $[6,10]$. The experiments are carried out on 33 problems, which are impartially chosen and divided into five test cases, to analyze the empirical results:

- The test case $T_{1}$ (see Sect. A.1) consists of eight easy problems with isolated solutions. These problems are solvable in short time by the search using all three propagators.

- The test case $T_{2}$ (see Sect. A.2) consists of four problems of moderate difficulty with isolated solutions. These problems are solvable by the search using FBPD and BOX and cause the search using HC4 being out of time without reaching $10^{6}$ splits.

- The test case $T_{3}$ (see Sect. A.3) consists of eight hard problems with isolated solutions. These problems cause the search using FBPD to stop due to running more than $10^{6}$ splits, cause the search using HC4 to be out of time without reaching $10^{6}$ splits, and cause the search using BOX either to be out of time or to stop due to running more than $10^{6}$ splits. $^{2}$

- The test case $T_{4}$ (see Sect. A.4) consists of seven easy problems with a continuum of solutions. These problems are solvable in short time at the predefined precision $10^{-2}$.

- The test case $T_{5}$ (see Sect. A.5) consists of six hard problems with a continuum of solutions. These problems are solvable in short time at the predefined precision $10^{-1}$.

The timeout value is set to $10 \mathrm{~h}$ for all the test cases. The timeout values will be used as the running time for the techniques that are out of time in the next result analysis favor of slow techniques. For the first three test cases, the precision is $10^{-4}$, and the search is done by bisection. For the last two test cases, the search is performed using the UCA6 [22], algorithm for inequalities. The comparison of the interval propagation techniques is based on the following measures:

- The running time: The relative ratio of the running time of each propagator to that of FBPD is called the relative time ratio.

\footnotetext{
2 FBPD essentially works at the node level. Evaluation/propagation procedures can therefore be run on selected nodes rather than on the entire graph. This enables the use of different interval forms at different steps of a propagation procedure. Such an approach was tested in [26]. This extension of FBPD was then able to solve 6 problems out of 8 in the test case $T_{3}$.
} 
- The number of boxes: The relative ratio of the number of boxes in the output of each propagator to that of FBPD is called the relative cluster ratio.

- The number of splits/iterations: The number of splits in search needed to solve the problems. The relative ratio of the number of splits used by each propagator to that of FBPD is called the relative iteration ratio.

- The volume of boxes ( only for $T_{1}, T_{2}, T_{3}$ ): We consider the reduction per dimension $\sqrt[d]{V / D}$; where $d$ is the dimension of the problem, $V$ is the total volume of the output boxes, $D$ is the volume of the initial domains. The relative ratio of the reduction gained by each propagator to that of FBPD is called the relative reduction ratio.

- The volume of inner boxes (only for $T_{4}, T_{5}$ ): The ratio of the volume of inner boxes to the volume of all output boxes is called the inner volume ratio.

The lower the relative ratio is, the better the performance/quality is; and the higher the inner volume ratio is, the better the quality is.

The overviews of results in our experiments are given in Tables 1 and 2.

In Table 3, we give the overrun ratio of each propagator for the test case $T_{1}$. The overrun ratio is defined as $\varepsilon / \sqrt[d]{V / N}$; where $\varepsilon$ is the required precision, $d$ is the dimension of the problem, $V$ is the total volume of the output boxes, $N$ is the number of output boxes.

Clearly, FBPD outperforms both BOX and HC4 by 1 to 2 orders of magnitude or more in speed. For problems with a continuum of solutions, FBPD has roughly the same quality with respect to enclosure properties.

For isolated solutions, very narrow boxes are produced by any technique in comparison to the required precision. However, the new technique is about 1.1-2.0 times less tight than the other techniques in the measure of reduction per dimension (which hardly matters in applications).

Table 1 A comparison of three constraint propagation techniques, FBPD, BOX and HC4, in solving NCSPs

\begin{tabular}{|c|c|c|c|c|c|c|c|c|}
\hline \multirow{2}{*}{$\begin{array}{l}\text { Propagator } \\
\nabla\end{array}$} & \multicolumn{4}{|c|}{ (a) Isolated solutions } & \multicolumn{4}{|c|}{ (b) Continuum of solutions } \\
\hline & $\begin{array}{l}\text { Relative } \\
\text { time } \\
\text { ratio }\end{array}$ & $\begin{array}{l}\text { Relative } \\
\text { reduction } \\
\text { ratio }\end{array}$ & $\begin{array}{l}\text { Relative } \\
\text { cluster } \\
\text { ratio }\end{array}$ & $\begin{array}{l}\text { Relative } \\
\text { iteration } \\
\text { ratio }\end{array}$ & $\begin{array}{l}\text { Relative } \\
\text { time } \\
\text { ratio }\end{array}$ & $\begin{array}{l}\text { Inner } \\
\text { volume } \\
\text { ratio }\end{array}$ & $\begin{array}{l}\text { Relative } \\
\text { cluster } \\
\text { ratio }\end{array}$ & $\begin{array}{l}\text { Relative } \\
\text { iteration } \\
\text { ratio }\end{array}$ \\
\hline FBPD & 1.000 & 1.000 & 1.000 & 1.000 & 1.000 & 0.922 & 1.000 & 1.000 \\
\hline BOX & 20.863 & 0.625 & 0.342 & 0.731 & 20.919 & 0.944 & 0.873 & 0.854 \\
\hline HC4 & 203.285 & 0.906 & 1.266 & 0.988 & 403.915 & 0.941 & 0.896 & 0.879 \\
\hline
\end{tabular}

In the section (a), the averages of the relative time ratios are taken over all the problems in the test cases $T_{1}, T_{2}, T_{3}$; and the averages of the other relative ratios are taken over the problems in the test case $T_{1}$. In the section (b), the averages of the relative ratios are taken over all the problems in the test cases $T_{4}, T_{5}$

Table 2 The averages of the relative time ratios are taken over the problems in each test case

\begin{tabular}{|c|c|c|c|c|c|}
\hline \multirow{2}{*}{ Propagator } & \multicolumn{3}{|c|}{ (a) Isolated solutions } & \multicolumn{2}{|c|}{ (b) Continuum of solutions } \\
\hline & Test case $T_{1}$ & Test case $T_{2}$ & Test case $T_{3}$ & Test case $T_{4}$ & Test case $T_{5}$ \\
\hline FBPD & 1.00 & 1.00 & 1.00 & 1.00 & 1.00 \\
\hline BOX & 24.21 & 28.98 & 13.45 & 11.55 & 31.85 \\
\hline HC4 & 94.42 & 691.24 & 68.17 & 191.86 & 651.31 \\
\hline
\end{tabular}


Table 3 The overrun ratios for the test case $T_{1}$

\begin{tabular}{llllllllll}
\hline Problem & BIF3 & REI3 & WIN3 & ECO5 & ECO6 & NEU6 & ECO7 & ECO8 & Average \\
\hline FBPD & 1.626 & 1.360 & 2.075 & 1.711 & 1.676 & 3.198 & 1.513 & 1.455 & $\mathbf{1 . 8 2 7}$ \\
BOX & 2.957 & 1.974 & 3.080 & 1.579 & 1.660 & 6.748 & 1.521 & 1.485 & $\mathbf{2 . 6 2 5}$ \\
HC4 & 2.229 & 1.914 & 1.492 & 1.647 & 1.679 & 4.949 & 1.488 & 1.449 & $\mathbf{2 . 1 0 6}$ \\
\hline
\end{tabular}

An overrun ratio greater than 1 would satisfy the requirements of applications

The gain in performance is more important for under-constrained problems than for wellconstrained ones.

\section{Conclusion}

We propose a new constraint propagation technique, called FBPD, which makes the fundamental framework of interval analysis on DAGs [21] efficient and practical for numerical constraint propagation. We also propose a method to coordinate constraint propagation (FBPD) and exhaustive search on partial DAG representations, where only one DAG for each problem is needed for the whole solution process. The experiments, carried out on various problems, show that the new approach can outperform previously available propagation techniques by 1 to 2 orders of magnitude or more in speed, while being roughly of same quality with respect to enclosure properties. Moreover, FBPD essentially works at the node level. Evaluation and propagation procedures can therefore be run on selected nodes rather than on the entire graph. This enables the use of different enclosure techniques at different steps of the propagation process opens up promising perspectives [26].

Acknowledgements The research was supported through the COCONUT project (MIST-2000-26063) by the European Commission and the Swiss Federal Education and Science Office (OFES), and through the FWF-Project P18704-N13 by the Austrian Science Foundation. We would like to thank ILOG for the software licenses of ILOG Solver used in the study, and also thank the COCONUT team of the University of Nantes for the HC4 source code. We also thank Professor Arnold Neumaier, University of Vienna (Austria), for his fruitful discussions and very valuable input.

\section{Appendix}

\section{A. Numerical benchmarks}

A.1 Test case $T_{1}$ : problems with isolated solutions

\section{A.1.1 Problem BIF3}

A bifurcation problem:

$$
\left\{\begin{array}{l}
5 x^{9}-6 x^{5} y^{2}+x y^{4}+2 x z=0 \\
-2 x^{6} y+2 x^{2} y^{3}+2 y z=0 \\
x^{2}+y^{2}=0.265625
\end{array}\right.
$$

where $x, y, z$ in $\left[-10^{8}, 10^{8}\right]$. 


\section{A.1.2 Problem ECO5}

An economic problem:

$$
\left\{\begin{array}{l}
\left(x_{1}+x_{1} x_{2}+x_{2} x_{3}+x_{3} x_{4}\right) x_{5}-1=0 \\
\left(x_{2}+x_{1} x_{3}+x_{2} x_{4}\right) x_{5}-2=0 \\
\left(x_{3}+x_{1} x_{4}\right) x_{5}-3=0 \\
x_{4} x_{5}-4=0 \\
x_{1}+x_{2}+x_{3}+x_{4}+1=0
\end{array}\right.
$$

where $x_{1}, \ldots, x_{5}$ in $[-10,10]$.

\section{A.1.3 Problem ECO6}

An economic problem:

$$
\left\{\begin{array}{l}
\left(x_{1}+x_{1} x_{2}+x_{2} x_{3}+x_{3} x_{4}+x_{4} x_{5}\right) x_{6}-1=0 \\
\left(x_{2}+x_{1} x_{3}+x_{2} x_{4}+x_{3} x_{5}\right) x_{6}-2=0 \\
\left(x_{3}+x_{1} x_{4}+x_{2} x_{5}\right) x_{6}-3=0 \\
\left(x_{4}+x_{1} x_{5}\right) x_{6}-4=0 \\
x_{5} x_{6}-5=0 \\
x_{1}+x_{2}+x_{3}+x_{4}+x_{5}+1=0
\end{array}\right.
$$

where $x_{1}, \ldots, x_{6}$ in $[-10,10]$.

\section{A.1.4 Problem ECO7}

An economic problem:

$$
\left\{\begin{array}{l}
\left(x_{1}+x_{1} x_{2}+x_{2} x_{3}+x_{3} x_{4}+x_{4} x_{5}+x_{5} x_{6}\right) x_{7}-1=0 \\
\left(x_{2}+x_{1} x_{3}+x_{2} x_{4}+x_{3} x_{5}+x_{4} x_{6}\right) x_{7}-2=0 \\
\left(x_{3}+x_{1} x_{4}+x_{2} x_{5}+x_{3} x_{6}\right) x_{7}-3=0 \\
\left(x_{4}+x_{1} x_{5}+x_{2} x_{6}\right) x_{7}-4=0 \\
\left(x_{5}+x_{1} x_{6}\right) x_{7}-5=0 \\
x_{6} x_{7}-6=0 \\
x_{1}+x_{2}+x_{3}+x_{4}+x_{5}+x_{6}+1=0
\end{array}\right.
$$

where $x_{1}, \ldots, x_{7}$ in $[-10,10]$.

\section{A.1.5 Problem ECO8}

An economic problem:

$$
\left\{\begin{array}{l}
\left(x_{1}+x_{1} x_{2}+x_{2} x_{3}+x_{3} x_{4}+x_{4} x_{5}+x_{5} x_{6}+x_{6} x_{7}\right) x_{8}-1=0 \\
\left(x_{2}+x_{1} x_{3}+x_{2} x_{4}+x_{3} x_{5}+x_{4} x_{6}+x_{5} x_{7}\right) x_{8}-2=0 ; \\
\left(x_{2}+x_{1} x_{3}+x_{2} x_{4}+x_{3} x_{5}+x_{4} x_{6}\right) x_{7}-2=0 ; \\
\left(x_{4}+x_{1} x_{5}+x_{2} x_{6}+x_{3} x_{7}\right) x_{8}-4=0 ; \\
\left(x_{5}+x_{1} x_{6}+x_{2} x_{7}\right) x_{8}-5=0 \\
\left(x_{6}+x_{1} x_{7}\right) x_{8}-6=0 \\
x_{7} x_{8}-7=0 \\
x_{1}+x_{2}+x_{3}+x_{4}+x_{5}+x_{6}+x_{7}+1=0 ;
\end{array}\right.
$$

where $x_{1}, \ldots, x_{8}$ in $[-10,10]$. 


\section{A.1.6 Problem NEU6}

A neurophysiology problem:

$$
\left\{\begin{array}{l}
x_{1}^{2}+x_{3}^{2}=1 \\
x_{2}^{2}+x_{4}^{2}=1 \\
x_{5} x_{1}^{3}+x_{6} x_{2}^{3}=5 \\
x_{5} x_{1} x_{3}^{2}+x_{6} x_{4}^{2} x_{2}=4 \\
x_{5} x_{3}^{3}+x_{6} x_{4}^{3}=3 \\
x_{5} x_{1}^{2} x_{3}+x_{6} x_{2}^{2} x_{4}=2 \\
x_{1} \geq x_{2} \\
x_{1} \geq 0 \\
x_{2} \geq 0
\end{array}\right.
$$

where $x_{1}, \ldots, x_{6}$ in $[-100,100]$.

\section{A.1.7 Problem REI3}

A neurophysiology problem:

$$
\left\{\begin{array}{l}
x^{2}-y^{2}+z^{2}=0.5 \\
x^{3}-y^{3}+z^{3}=0.5 \\
x^{4}-y^{4}+z^{4}=0.5 \\
2 x y+6 y^{2}+2 y z-2 x-4 y-2 z+1=0
\end{array}\right.
$$

where $x, y, z$ in $[-10,10]$.

\section{A.1.8 Problem WIN3}

A neurophysiology problem:

$$
\left\{\begin{array}{l}
4 x z-4 x y^{2}-16 x^{2}-1=0 \\
2 y^{2} z+4 x+1=0 \\
2 x^{2} z+2 y^{2}+x=0 \\
2 x y+6 y^{2}+2 y z-2 x-4 y-2 z+1=0
\end{array}\right.
$$

where $x, y, z$ in $\left[-10^{5}, 10^{5}\right]$.

A.2 Test case $T_{2}$ : problems with isolated solutions

\section{A.2.1 Problem CYC5}

A cyclic problem:

$$
\left\{\begin{array}{l}
a+b+c+d+e=0 \\
a b+b c+c d+d e+e a=0 \\
a b c+b c d+c d e+d e a+e a b=0 \\
a b c d+b c d e+c d e a+d e a b+e a b c=0 \\
a b c d e-1=0 ;
\end{array}\right.
$$

where $a, b, c, d, e$ in $[-10,10]$. 


\section{A.2.2 Problem GS5.1}

A Gough Steward problem:

$$
\left\{\begin{array}{l}
x_{1}^{2}+y_{1}^{2}+z_{1}^{2}=31, \\
x_{2}^{2}+y_{2}^{2}+z_{2}^{2}=39, \\
x_{3}^{2}+y_{3}^{2}+z_{3}^{2}=29, \\
x_{1} x_{2}+y_{1} y_{2}+z_{1} z_{2}+6 x_{1}-6 x_{2}=51, \\
x_{1} x_{3}+y_{1} y_{3}+z_{1} z_{3}+7 x_{1}-2 y_{1}-7 x_{3}+2 y_{3}=50, \\
x_{2} x_{3}+y_{2} y_{3}+z_{2} z_{3}+x_{2}-2 y_{2}-x_{3}+2 y_{3}=34, \\
-12 x_{1}+15 y_{1}-10 x_{2}-25 y_{2}+18 x_{3}+18 y_{3}=-32, \\
-14 x_{1}+35 y_{1}-36 x_{2}-45 y_{2}+30 x_{3}+18 y_{3}=8, \\
2 x_{1}+2 y_{1}-14 x_{2}-2 y_{2}+8 x_{3}-y_{3}=20,
\end{array}\right.
$$

where $x_{1} \in[0.00 ; 5.57], y_{1} \in[0.00,2.70], z_{1} \in[0.00,5.57], x_{2} \in[-6.25,0.00], y_{2} \in$ $[-2.00,0.00], z_{2} \in[0.00,6.25], x_{3} \in[-5.39,-1.00], y_{3} \in[-5.39,0.00], z_{3} \in[0.00,5.39]$.

\section{A.2.3 Problem KOL2}

Kolev's benchmark:

$$
\left\{\begin{array}{l}
\left(\left(4 x_{3}+3 x_{6}\right) x_{3}+2 x_{5}\right) x_{3}+x_{4}=0 \\
\left(\left(4 x_{2}+3 x_{6}\right) x_{2}+2 x_{5}\right) x_{2}+x_{4}=0 \\
\left(\left(4 x_{1}+3 x_{6}\right) x_{1}+2 x_{5}\right) x_{1}+x_{4}=0 \\
x_{4}+x_{5}+x_{6}+1=0 \\
\left(\left(\left(x_{2}+x_{6}\right) x_{2}+x_{5}\right) x_{2}+x_{4}\right) x_{2}+\left(\left(\left(x_{3}+x_{6}\right) x_{3}+x_{5}\right) x_{3}+x_{4}\right) x_{3}=0, \\
\left(\left(\left(x_{1}+x_{6}\right) x_{1}+x_{5}\right) x_{1}+x_{4}\right) x_{1}+\left(\left(\left(x_{2}+x_{6}\right) x_{2}+x_{5}\right) x_{2}+x_{4}\right) x_{3}=0,
\end{array}\right.
$$

where $x_{1} \in[0.0333,0.2173], x_{2} \in[0.4000,0.6000], x_{3} \in[0.7826,0.9666], x_{4} \in[-0.3071$, $-0.1071], x_{5} \in[1.1071,1.3071], x_{6} \in[-2.1000,-1.9000]$.

\section{A.2.4 Problem YAM60}

The Yama160 problem:

$$
(n+1)^{2} x_{i-1}-2(n+1)^{2} x_{i}+(n+1)^{2} x_{i+1}+e^{x_{i}}=0, \quad(\text { for } i=1, \ldots, n),
$$

where $n=60, x_{0}=x_{n+1}=0$, and $x_{i} \in[-10,10]$ (for $\left.i=1, \ldots, n\right)$,

A.3 Test case $T_{3}$ : problems with isolated solutions

\section{A.3.1 Problem CAP4}

A Caprasse problem:

$$
\left\{\begin{array}{l}
y^{2} z+2 x y t-2 x-z=0 \\
-x^{3} z+4 x y^{2} z+4 x^{2} y t+2 y^{3} t+4 x^{2}-10 y^{2}+4 x z-10 y t+2=0 \\
2 y z t+x t^{2}-x-2 z=0 \\
-x z^{3}+4 y z^{2} t+4 x z t^{2}+2 y t^{3}+4 x z+4 z^{2}-10 y t-10 t^{2}+2=0
\end{array}\right.
$$

where $x, y, z, t$ in $\mathbb{R}$. 


\section{A.3.2 Problem DID9}

A Didrit problem:

$$
\left\{\begin{array}{l}
x_{1}^{2}+y_{1}^{2}+z_{1}^{2}=31 \\
x_{2}^{2}+y_{2}^{2}+z_{2}^{2}=39 \\
x_{3}^{2}+y_{3}^{2}+z_{3}^{2}=29 \\
x_{1} x_{2}+y_{1} y_{2}+z_{1} z_{2}+6 x_{1}-6 x_{2}=51 \\
x_{1} x_{3}+y_{1} y_{3}+z_{1} z_{3}+7 x_{1}-2 y_{1}-7 x_{3}+2 y_{3}=50 \\
x_{2} x_{3}+y_{2} y_{3}+z_{2} z_{3}+x_{2}-2 y_{2}-x_{3}+2 y_{3}=34 ; \\
-12 x_{1}+15 y_{1}-10 x_{2}-25 y_{2}+18 x_{3}+18 y_{3}=-32 \\
-14 x_{1}+35 y_{1}-36 x_{2}-45 y_{2}+30 x_{3}+18 y_{3}=8 ; \\
2 x_{1}+2 y_{1}-14 x_{2}-2 y_{2}+8 x_{3}-y_{3}=20
\end{array}\right.
$$

where $x_{i}, y_{i}, z_{i}$ in $[-10,10]$ for $i=1,2,3$.

\section{A.3.3 Problem GS5.0}

A Gough Steward problem:

$$
\left\{\begin{array}{l}
x_{1}^{2}+y_{1}^{2}+z_{1}^{2}=31 \\
x_{2}^{2}+y_{2}^{2}+z_{2}^{2}=39 \\
x_{3}^{2}+y_{3}^{2}+z_{3}^{2}=29 \\
x_{1} x_{2}+y_{1} y_{2}+z_{1} z_{2}+6 x_{1}-6 x_{2}=51 \\
x_{1} x_{3}+y_{1} y_{3}+z_{1} z_{3}+7 x_{1}-2 y_{1}-7 x_{3}+2 y_{3}=50 \\
x_{2} x_{3}+y_{2} y_{3}+z_{2} z_{3}+x_{2}-2 y_{2}-x_{3}+2 y_{3}=34 \\
-12 x_{1}+15 y_{1}-10 x_{2}-25 y_{2}+18 x_{3}+18 y_{3}=-32 \\
-14 x_{1}+35 y_{1}-36 x_{2}-45 y_{2}+30 x_{3}+18 y_{3}=8 \\
2 x_{1}+2 y_{1}-14 x_{2}-2 y_{2}+8 x_{3}-y_{3}=20
\end{array}\right.
$$

where $x_{1} \in[-2.00 ; 5.57], y_{1} \in[-5.57,2.70], z_{1} \in[0.00,5.57], x_{2} \in[-6.25,1.30]$, $y_{2} \in[-6.25,2.70], z_{2} \in[-2.00,6.25], x_{3} \in[-5.39,0.70], y_{3} \in[-5.39,3.11], z_{3} \in$ $[-3.61,5.39]$.

\section{A.3.4 Problem KAT8}

A Katsura problem:

$$
\left\{\begin{array}{l}
-x_{1}+2 x_{8}^{2}+2 x_{7}^{2}+2 x_{6}^{2}+2 x_{5}^{2}+2 x_{4}^{2}+2 x_{3}^{2}+2 x_{2}^{2}+x_{1}^{2}=0 \\
-x_{2}+2 x_{8} x_{7}+2 x_{7} x_{6}+2 x_{6} x_{5}+2 x_{5} x_{4}+2 x_{4} x_{3}+2 x_{3} x_{2}+2 x_{2} x_{1}=0 \\
-x_{3}+2 x_{8} x_{6}+2 x_{7} x_{5}+2 x_{6} x_{4}+2 x_{5} x_{3}+2 x_{4} x_{2}+2 x_{3} x_{1}+x_{2}^{2}=0 \\
-x_{4}+2 x_{8} x_{5}+2 x_{7} x_{4}+2 x_{6} x_{3}+2 x_{5} x_{2}+2 x_{4} x_{1}+2 x_{3} x_{2}=0 \\
-x_{5}+2 x_{8} x_{4}+2 x_{7} x_{3}+2 x_{6} x_{2}+2 x_{5} x_{1}+2 x_{4} x_{2}+x_{3}^{2}=0 \\
-x_{6}+2 x_{8} x_{3}+2 x_{7} x_{2}+2 x_{6} x_{1}+2 x_{5} x_{2}+2 x_{4} x_{3}=0 \\
-x_{7}+2 x_{8} x_{2}+2 x_{7} x_{1}+2 x_{6} x_{2}+2 x_{5} x_{3}+x_{4}^{2}=0 \\
-1+2 x_{8}+2 x_{7}+2 x_{6}+2 x_{5}+2 x_{4}+2 x_{3}+2 x_{2}+x_{1}=0
\end{array}\right.
$$

where $x_{1}, \ldots, x_{8}$ in $[-10,10]$. 


\section{A.3.5 Problem KIN9}

A kinematics problem:

$$
\left\{\begin{array}{l}
z_{1}^{2}+z_{2}^{2}+z_{3}^{2}-12 z_{1}-68=0 \\
z_{4}^{2}+z_{5}^{2}+z_{6}^{2}-12 z_{5}-68=0 \\
z_{7}^{2}+z_{8}^{2}+z_{9}^{2}-24 z_{8}-12 z_{9}+100=0 \\
z_{1} z_{4}+z_{2} z_{5}+z_{3} z_{6}-6 z_{1}-6 z_{5}-52=0 \\
z_{1} z_{7}+z_{2} z_{8}+z_{3} z_{9}-6 z_{1}-12 z_{8}-6 z_{9}+64=0 \\
z_{4} z_{7}+z_{5} z_{8}+z_{6} z_{9}-6 z_{5}-12 z_{8}-6 z_{9}+32=0 \\
2 z_{2}+2 z_{3}-z_{4}-z_{5}-2 z_{6}-z_{7}-z_{9}+18=0 \\
z_{1}+z_{2}+2 z_{3}+2 z_{4}+2 z_{6}-2 z_{7}+z_{8}-z_{9}-38=0 \\
z_{1}+z_{3}-2 z_{4}+z_{5}-z_{6}+2 z_{7}-2 z_{8}+8=0
\end{array}\right.
$$

where $z_{1}, \ldots, z_{9}$ in $[-1000,1000]$.

\section{A.3.6 Problem REI4}

A Reinmer system:

$$
\left\{\begin{array}{l}
x^{2}-y^{2}+z^{2}-t^{2}=0.5 \\
x^{3}-y^{3}+z^{3}-t^{3}=0.5 \\
x^{4}-y^{4}+z^{4}-t^{4}=0.5 \\
x^{5}-y^{5}+z^{5}-t^{5}=0.5
\end{array}\right.
$$

where $x, y, z, t$ in $[-10,10]$.

\section{A.3.7 Problem REI5}

A Reinmer system:

$$
\left\{\begin{array}{l}
-1+2 x_{1}^{2}-2 x_{2}^{2}+2 x_{3}^{2}-2 x_{4}^{2}+2 x_{5}^{2}=0 \\
-1+2 x_{1}^{3}-2 x_{2}^{3}+2 x_{3}^{3}-2 x_{4}^{3}+2 x_{5}^{3}=0 \\
-1+2 x_{1}^{4}-2 x_{2}^{4}+2 x_{3}^{4}-2 x_{4}^{4}+2 x_{5}^{4}=0 \\
-1+2 x_{1}^{5}-2 x_{2}^{5}+2 x_{3}^{5}-2 x_{4}^{5}+2 x_{5}^{5}=0 \\
-1+2 x_{1}^{6}-2 x_{2}^{6}+2 x_{3}^{6}-2 x_{4}^{6}+2 x_{5}^{6}=0
\end{array}\right.
$$

where $x_{1}, \ldots, x_{5}$ in $[-1,1]$.

\section{A.3.8 Problem REI6}

A Reinmer system:

$$
\left\langle-0.5+\sum_{i=1}^{n}(-1)^{i+1} x_{i}^{k}=0(k=1, \ldots, n) ; n=6, x_{i} \in[-1,1](\text { for } i=1, \ldots, n)\right\rangle
$$


A.4 Test case $T_{4}$ : problems with continuums of solutions

\section{A.4.1 Problem $\mathbf{F} 2.2$}

Tricuspoid and Circle:

$$
\left\{\begin{array}{l}
\left(x^{2}+y^{2}+12 x+9\right)^{2} \leq 4(2 x+3)^{3} \\
x^{2}+y^{2} \geq 2
\end{array}\right.
$$

where $x, y$ in $[-2,2]$.

\section{A.4.2 Problem $\mathbf{F 2 . 3}$}

Foliumd, Circle, and Trifolium:

$$
\left\{\begin{array}{l}
x^{3}+y^{3} \geq 3 x y \\
x^{2}+y^{2} \geq 0.1 \\
\left(x^{2}+y^{2}\right)\left(y^{2}+x(x+1)\right) \leq 4 x y^{2}
\end{array}\right.
$$

where $x, y$ in $[-3,3]$.

\section{A.4.3 Problem $\mathbf{S 0 4}$}

Circle:

$$
\left\langle x^{2}+y^{2} \leq 1 ; x, y \in[-2,2]\right\rangle
$$

\section{A.4.4 Problem $\mathbf{S 0 5}$}

$$
\left\langle\frac{x}{\sqrt{(y-5)^{2}+1}} \leq 1 ; x, y \in[1,10]\right\rangle
$$

\section{A.4.5 Problem $\mathbf{S 0 6}$}

$$
\left\langle\frac{12 y}{\sqrt{(x-12)^{2}+y^{2}}} \leq 10 ; x \in[-50,50], y \in[0,50]\right\rangle
$$

\section{A.4.6 Problem $\mathbf{S 0 7}$}

$$
\left\langle x^{2}+y^{2} \geq 20 ; x^{2}+y^{2} \leq 50 ; x \in[-50,50], y \in[0,50]\right\rangle
$$

\section{A.4.7 Problem WP}

A Kinematic Pair (of a wheel and a pawl):

$$
\left\langle 20 \leq \sqrt{x^{2}+y^{2}} \leq 50, \frac{12 y}{\sqrt{(x-12)^{2}+y^{2}}} \leq 10 ; x \in[-50,50], y \in[0,50]\right\rangle
$$


A.5 Test case $T_{5}$ : problems with continuums of solutions

\section{A.5.1 Problem G1.1}

$$
\left\{\begin{array}{l}
x_{1}^{2}+0.5 x_{2}+2\left(x_{3}-6\right) \geq 0 \\
x_{1}^{2}+x_{2}^{2}+x_{3}^{2} \leq 25
\end{array}\right.
$$

where $x_{1}, x_{2}, x_{3}$ in $[-8,8]$.

\section{A.5.2 Problem G1.1}

$$
\left\{\begin{array}{l}
x_{1}^{2}+0.5 x_{2}+2\left(x_{3}-3\right) \geq 0 \\
x_{1}^{2}+x_{2}^{2}+x_{3}^{2} \leq 25
\end{array}\right.
$$

where $x_{1}, x_{2}, x_{3}$ in $[-8,8]$.

\section{A.5.3 Problem H1.1}

$$
\left\{\begin{array}{l}
x_{1}^{2}+x_{2}^{2}+x_{3}^{2} \leq 9 \\
\left(x_{1}-0.5\right)^{2}+\left(x_{2}-1\right)^{2}+x_{3}^{2} \geq 4 \\
x_{1}^{2}+\left(x_{2}-0.2\right)^{2} \geq x_{3}
\end{array}\right.
$$

where $x_{1}, x_{2}, x_{3}$ in $[-4,4]$.

\section{A.5.4 Problem P1.4}

$$
\left\{\begin{array}{l}
x^{2}+y^{2}+z^{2}<=4 \\
(x-2)^{2}+y^{2}+z^{2}>=4
\end{array}\right.
$$

where $x, y, z$ in $[-4,4]$.

\section{A.5.5 Problem $\mathbf{P 2}$}

$$
\left\{\begin{array}{l}
x^{2} \leq y \\
\ln y+1 \geq z \\
x z \leq 1
\end{array}\right.
$$

where $x \in[0,15], y \in[1,200], z \in[-10,10]$.

\section{A.5.6 Problem $\mathbf{P 3}$}

$$
\left\{\begin{array}{l}
x^{2} \leq y \\
\ln y+1 \geq z \\
x z \leq 1 \\
x^{3 / 2}+\ln (1.5 z+1) \leq y+1
\end{array}\right.
$$

where $x \in[0,15], y \in[1,200], z \in[0,10]$. 


\section{References}

1. Alefeld, G., Herzberger, J.: Introduction to Interval Computations. Academic Press, New York (1983)

2. Benhamou, F., Older, W.J.: Applying interval arithmetic to real, integer and boolean constraints. J. Log. Programm, pp. 32-81, 1997. Extension of a Technical Report of Bell Northern Research, Canada (1992)

3. Benhamou, F., Older, W.J.: Applying interval arithmetic to real, integer and boolean constraints. Technical Report BNR, Technical Report, Bell Northern Research, ON, Canada (1992)

4. Benhamou, F., McAllester, D., Van Hentenryck, P.: CLP(Intervals) revisited. In: Proceedings of the International Logic Programming Symposium, pp. 109-123 (1994)

5. Benhamou, F., Goualard, F., Granvilliers, L., Puget, J.-F.: Revising hull and box consistency. In: Proceedings of the International Conference on Logic Programming (ICLP'99), pp. 230-244. Las Cruces, USA (1999)

6. Granvilliers, L., Benhamou, F.: Algorithm 852: RealPaver: an interval solver using constraint satisfaction techniques. ACM Trans. Math. Softw. (TOMS) 32(1), 138-156 (2006)

7. Hansen, E.R., Walster, G.W.: Global optimization using interval analysis, 2nd edn. Marcel Dekker (2004)

8. Hickey, T.J., Ju, Q., Van Emden, M.H.: Interval arithmetic: from principles to implementation. J. ACM (JACM) 48(5), 1038-1068 (2001)

9. Jaulin, L., Kieffer, M., Didrit, O., Walter, E.: Applied interval analysis, 1st edn. Springer (2001)

10. Lebbah, Y.: ICOS (Interval Constraints Solver). WWW document (2003)

11. Lhomme, O.: Consistency techniques for numeric CSPs. In: Proceedings of the 13th International Joint Conference on Artificial Intelligence (IJCAI-93), pp. 232-238 (1993)

12. Lottaz, C.: Collaborative design using solution spaces. PhD thesis, Swiss Federal Institute of Technology in Lausanne (EPFL), Switzerland (2000)

13. Mackworth, A.K.: Consistency in networks of relations. Artif. Intell. 8, 99-118 (1977)

14. Montanari, U.: Networks of constraints: fundamental properties and applications to picture processing. Inf. Sci. 7, 95-132 (1974)

15. Moore, R.E.: Interval Analysis. Prentice Hall, Englewood Cliffs, NJ (1966)

16. Moore, R.E.: Methods and Applications of Interval Analysis. SIAM Studies in Applied Mathematics, Philadelphia (1979)

17. Neumaier, A.: Interval Methods for Systems of Equations. Cambridge University Press, Cambridge (1990)

18. Pryce, J.D., Corliss, G.F.: Interval arithmetic with containment sets. Computing 78(3), 251-276 (2006)

19. Sam-Haroud, D.: Constraint consistency techniques for continuous domains. PhD thesis, Swiss Federal Institute of Technology in Lausanne (EPFL), Switzerland (1995)

20. Schichl, H.: Mathematical modeling and global optimization. Habilitation thesis, Faculty of Mathematics, University of Vienna, Autralia, November (2003)

21. Schichl, H., Neumaier, A.: Interval analysis on directed acyclic graphs for global optimization. J. Global Optim. 33, 541-562 (2005)

22. Silaghi, M.-C., Sam-Haroud, D., Faltings, B.: Search techniques for non-linear CSPs with inequalities. In: Proceedings of the 14th Canadian Conference on Artificial Intelligence (2001)

23. Singh, S., Watson, B., Srivastava, P.: Fixed point theory and best approximation: the KKM-map principle. Kluwer Academic Publishers, Dordrecht (1997)

24. Van Hentenryck, P.: Numerica: a modeling language for global optimization. In: Proceedings of the 15 th International Joint Conference on Artificial Intelligence (IJCAI-97) (1997)

25. Vu, X.-H., Sam-Haroud, D., Silaghi, M.-C.: Numerical constraint satisfaction problems with non-isolated solutions. In: Global Optimization and Constraint Satisfaction: First International Workshop on Global Constraint Optimization and Constraint Satisfaction, COCOS 2002, LNCS vol. 2861, pp. 194-210. Valbonne-Sophia Antipolis, France, October 2003. Springer-Verlag

26. Vu, X.-H., Sam-Haroud, D., Faltings, B.: Combining multiple inclusion representations in numerical constraint propagation. In: Proceedings of the 16th IEEE International Conference on Tools with Artificial Intelligence (ICTAI 2004), pp. 458-467. Florida, USA, November 2004. IEEE Computer Society Press

27. Vu, X.-H., Schichl, H., Sam-Haroud, D.: Using directed acyclic graphs to coordinate propagation and search for numerical constraint satisfaction problems. In: Proceedings of the 16th IEEE International Conference on Tools with Artificial Intelligence (ICTAI 2004), Florida, USA, November 2004. IEEE Computer Society Press

28. Waltz, D.L.: Generating semantic descriptions from drawings of scenes with shadows. Technical Report, Massachusetts Institute of Technology, USA (1972)

29. Waltz, D.L.: The Psychology of Computer Vision, Chapter Understanding Line Drawings of Scenes with Shadows, pp. 19-91. McGraw Hill, New York (1975) 
30. William Walster, G., Hansen, E.R., Pryce, J.D.: Extended real intervals and the topological closure of extended real relations. Technical Report, Sun Microsystems, February (2000). http://wwws.sun.com/ software/sundev/whitepapers/extended-real.pdf 